\title{
Chern Currents of Singular Connections Associated with a Section of a Compactified Bundle
}

\section{JOHN ZWECK}

\begin{abstract}
A compactification of the Chern-Weil theory for bundle maps, developed in [HL], is described. For each section $\nu$ of the compactification $\mathbb{P}(\underline{\mathbb{C}} \oplus F) \rightarrow X$ of a rank $n$ complex vector bundle $F \rightarrow X$ with connection, and for each Adinvariant polynomial $\phi$ on $\mathfrak{g l}_{n}$, there is an associated current formula

$$
\begin{array}{r}
\phi\left(\Omega_{F}\right)+\nu^{*}\left(\operatorname{Res}_{\infty}(\phi)\right) \operatorname{Div}_{\infty}(\nu)-\phi\left(\Omega_{0}\right)-\operatorname{Res}_{0}(\phi) \operatorname{Div}_{0}(\nu) \\
=d T \text { on } X,
\end{array}
$$
\end{abstract}

generalizing that of [HL]. Here $\operatorname{Div}_{0}(\nu)$ and $\operatorname{Div}_{\infty}(\nu)$ are integrally flat currents supported on the zero and pole sets of $\nu$, $\operatorname{Res}_{0}(\phi)$ and $\operatorname{Res}_{\infty}(\phi)$ are smooth residue forms which can be calculated in terms of the curvature $\Omega_{F}$ of $F, T$ is a canonical transgression form with coefficients in $L_{\text {loc }}^{1}(X)$, and $\phi\left(\Omega_{0}\right)$ is an $L_{\text {loc }}^{1}(X)$ form canonically defined in terms of a singular connection naturally associated to $\nu$.

This result holds for $C^{\infty}$-meromorphic sections $\nu$ which are atomic. The notion of an atomic section of a vector bundle was first introduced and studied in [HS]. The formulae obtained include a generalization of the Poincaré-Lelong formula to $C^{\infty}$-meromorphic sections of a bundle of arbitrary rank.

Introduction. The main result of this paper is a generalization of the following version of the argument principle for smooth maps from a manifold into complex projective space. 
The argument principle. Let $H$ be a copy of $\mathbb{C P}^{n-1}$ in complex projective space $\mathbb{C P}^{n}$, and let $q$ be a point in $\mathbb{C P}^{n} \sim H \cong \mathbb{C}^{n}$. Let $X$ be a compact oriented real $2 n$-dimensional smooth manifold with boundary $\partial X$, and let $f: X \rightarrow \mathbb{C P}^{n}$ be a smooth map. Suppose that $f^{-1}(q)=\left\{z_{1}, \ldots, z_{N}\right\}$ and (for the sake of simplicity) that $P=f^{-1}(H)$ is a smooth oriented connected $2(n-1)$ dimensional submanifold of $X$. Suppose that $\left(f^{-1}(q) \cup P\right) \cap \partial X=\varnothing$. Then

$$
\sum_{i=1}^{N} \operatorname{Mult}\left(f, z_{i}\right)-\operatorname{Deg}\left(\left.f\right|_{P}\right) \operatorname{Mult}(f, P)=\operatorname{Wind}(f, \partial X) .
$$

Here $\operatorname{Deg}\left(\left.f\right|_{P}\right)$ is the degree of the mapping $f: P \rightarrow H$. Let $\pi: \mathbb{C}^{n} \sim\{q\} \rightarrow$ $S^{2 n-1}$ be radial projection. Then $\operatorname{Mult}\left(f, z_{i}\right):=\operatorname{Deg}\left(\pi \circ f: \partial B\left(z_{i}\right) \rightarrow S^{2 n-1}\right)$ where $B\left(z_{i}\right)$ is a ball about $z_{i}$ not containing any other points of $f^{-1}(q) \cup P$. To define $\operatorname{Mult}(f, P)$, note that, for each $x \in P, f$ defines a map from the normal plane $N_{x} P$ to $N_{f(x)} H$. Then, for almost any $x \in P, \operatorname{Mult}(f, P)$ is the degree of the induced map between circles. Finally, the winding number, Wind $(f, \partial X)$, of $f$ around $\partial X$ is defined to be the sum of the degrees of the induced maps from the various connected components of $\partial X$ to the unit sphere in $\mathbb{C}^{n} \cong \mathbb{C P}^{n} \sim H$.

This well-known result is an immediate consequence of a $C^{\infty}$ generalization to arbitrary rank vector bundles of the Poincaré-Lelong equation of complex analysis, which is derived in this paper. The generalized Poincaré-Lelong equation is in turn a compactification of a Chern current formula derived by Harvey and Lawson in their beautiful paper " A theory of characteristic currents associated with a singular connection", [HL] (also see [HL-A]). Their theory associates canonical current representatives of the characteristic classes of a vector bundle to sections of that bundle. In this paper, we generalize their work by computing the currents associated to " $C^{\infty}$-meromorphic" sections of a vector bundle. The analytic underpinning for [HL], and for the results described here, is given in detail in Harvey and Semmes' paper "Zero divisors of atomic functions", [HS].

Let $F \rightarrow X$ be a rank $n$ complex vector bundle over an oriented manifold, and let $\phi$ be an $\mathrm{Ad}$-invariant polynomial on $\mathfrak{g l}(n, \mathbb{C})$. For each choice of connection on $F$ the Chern-Weil theory constructs a $d$-closed differential form $\phi\left(\Omega_{F}\right)$ on $X$, called the $\phi$-Chern form, which represents the $\phi$-Chern class of $F$ and is defined in terms of the curvature of the connection. Harvey and Lawson generalize this theory by associating to each sufficiently well-behaved smooth section $\alpha$ of the vector bundle $F$ with connection, a canonically defined $d$-closed current $\phi\left(\Omega_{F, \alpha}\right)$, called the $\phi$-Chern current of $\alpha$, which also represents the $\phi$-Chern class of $F$. This current can be expressed as the sum of an absolutely continuous part and a singular part which is supported on the zero set of the section $\alpha$. They 
also construct a canonically defined transgression current $T=T(\phi, \alpha)$ satisfying the current equation

$$
\phi\left(\Omega_{F, \alpha}\right)-\phi\left(\Omega_{F}\right)=d T \quad \text { on } X .
$$

The most interesting example is obtained by choosing the invariant polynomial to be the top Chern polynomial $c_{n}=(i / 2 \pi)^{n}$ det. Then, if 0 is a regular value of $\alpha$, the Chern current $c_{n}\left(\Omega_{F, \alpha}\right)$ is equal to the current $[Z]$ of integration over the zero set $Z$ of $\alpha$, and we have the fundamental current equation,

$$
[Z]-c_{n}\left(\Omega_{F}\right)=d T
$$

on $X$.

In this paper we study the $\phi$-Chern currents associated to "meromorphic" sections of a complex vector bundle $F$. By a meromorphic section of $F$ we mean a $C^{\infty}$-section $\nu$ of the bundle $\mathbb{P}(\underline{\mathbb{C}} \oplus F) \rightarrow X$ of projective spaces which compactifies the bundle $F \rightarrow X$. The pole set of such a section $\nu$ is the subset $P$ of $X$ defined by $P:=\nu^{-1}(\mathbb{P}(\underline{\mathbb{C}} \oplus F) \sim F)$. In analogy with Harvey and Lawson's work, we associate to $\nu$ a canonically defined current $\phi\left(\Omega_{F, \nu}\right)$ which also represents the $\phi$-Chern class of $F$ and whose singular support is contained in the union of the zero and pole sets of $\nu$. On the complement of the pole set the meromorphic section $\nu$ defines a smooth section $\alpha$ of $F$ and the current $\phi\left(\Omega_{F, \nu}\right)$ is equal to Harvey and Lawson's current $\phi\left(\Omega_{F, \alpha}\right)$. The main aim of this paper is to study the current $\phi\left(\Omega_{F, \nu}\right)$ in a neighbourhood of the pole set $P$. For instance, in the special case that the zero and pole sets of $\nu$ do not intersect the critical set of the section $\nu$, the $c_{n}$-Chern current of $\nu$ is of the form

$$
c_{n}\left(\Omega_{F, \nu}\right)=[Z]-\operatorname{Res}[P]
$$

on $X$,

where $[P]$ denotes the current of integration over the pole set $P$ and Res is a $d$-closed smooth form. Furthermore, as a generalization of $(\star \star)$, we derive a current equation of the form

$$
[Z]-\operatorname{Res}[P]-c_{n}\left(\Omega_{F}\right)=d T
$$

on $X$.

This equation is a special case of a current equation which generalizes the Poincaré-Lelong formula of complex analysis. Note that the classical PoincaréLelong formula only holds for meromorphic sections of holomorphic line bundles, whereas the generalizations described in this paper hold for $C^{\infty}$-meromorphic sections of smooth complex vector bundles of arbitrary rank.

Choosing $F$ to be the the trivial bundle corresponds to the study of maps $f: X \rightarrow \mathbb{C P}^{n}$. Suppose that $f$ satisfies the conditions given in the statement of the argument principle above. Then we have the current equation

$$
\sum_{i=1}^{N} \operatorname{Mult}\left(f, z_{i}\right)\left[z_{i}\right]-\operatorname{Mult}(f, P)[P] f^{*}(\mathrm{Vol})=d T
$$


where Vol is the volume form on $H$ in the Fubini-Study metric. The argument principle is obtained by letting the terms in this equation act on the test function $[X]$ on $X$.

We begin by describing Harvey and Lawson's construction of the Chern currents associated to a bundle map. Let $E$ and $F$ be complex vector bundles of ranks 1 and $n$ defined over a smooth oriented manifold $X$. Endow $E$ and $F$ with connections $D_{E}$ and $D_{F}$ and let $E \stackrel{\alpha}{\rightarrow} F$ be a smooth bundle map with zero set $Z=\left\{x \in X: \alpha_{x} \equiv 0\right\}$. (Note that when $E$ is the trivial bundle $\underline{\mathbb{C}}$, a bundle map $\alpha$ defines a section $\mu$ of $F$ by $\alpha(1)=\mu$.) Harvey and Lawson use the bundle map $\alpha$ to pushforward the connection $D_{E}$ on $E$ to a smooth connection $\vec{D}$ defined on $F$ over $X \sim Z$ (see Definition 2.3). This connection is called the singular pushforward connection associated with $\alpha$. It provides a way to relate the curvature operators of the bundles $E$ and $F$ to the zero set of the map $E \stackrel{\alpha}{\rightarrow} F$. This relationship is made explicit by computing "Chern currents" for $\vec{D}$.

Let $\phi$ be an Ad-invariant polynomial on $\mathfrak{g l}(n, \mathbb{C})$. The $\phi$-Chern current of $\vec{D}$ at time zero, $\phi\left(\left(\vec{D}_{0}\right)\right)$, on $X$ is defined as follows. First, Harvey and Lawson construct (see Definition 2.6) a smooth family $\vec{D}_{s}$, for $0<s<\infty$, of smooth connections on $F$ over all of $X$ so that as $s \rightarrow 0, \vec{D}_{s} \rightarrow \vec{D}$ on $F$ over $X \sim Z$ and as $s \rightarrow \infty, \vec{D}_{s} \rightarrow D_{F}$ on $F$ over $X$. Then the Chern current at time zero on $X$ is defined to be the current limit

$$
\phi\left(\left(\vec{D}_{0}\right)\right):=\lim _{s \rightarrow 0} \phi\left(\vec{D}_{s}\right)
$$

on $X$,

whenever this limit exists. Actually, there are many families $\vec{D}_{s}$ which have the required limits as $s \rightarrow 0$ and $s \rightarrow \infty$. We choose to work with the particular family $\vec{D}_{s}$ defined above because it arises naturally from a geometric construction to be described below.

In order that the Chern current $\phi\left(\left(\vec{D}_{0}\right)\right)$ exist it is necessary to impose some conditions on the behaviour of the map $\alpha$ near its zero set. To that end Harvey and Semmes defined the notion of an atomic bundle map $\alpha$ and defined the zero divisor, $\operatorname{Div}_{0}(\alpha)$, of such a map. The zero divisor is an integrally flat current supported on the zero set of $\alpha$ which encodes the multiplicity of vanishing of $\alpha$. Atomicity is a weak condition which ensures the existence of a zero divisor.

Harvey and Lawson proved that, if the bundle map $\alpha$ is atomic, the Chern current $\phi\left(\left(\vec{D}_{0}\right)\right)$ exists and is of the form

$$
\phi\left(\left(\vec{D}_{0}\right)\right)=\phi\left(\vec{D}_{0}\right)+\operatorname{Res}_{\phi}\left(\vec{D}_{0}\right) \operatorname{Div}_{0}(\alpha) \quad \text { on } X
$$


where $\phi\left(\vec{D}_{0}\right)$ is a $d$-closed $L_{\text {loc }}^{1}$ form on $X$ which agrees with the the smooth form $\phi(\vec{D})$ on $X \sim Z$, and the residue form, $\operatorname{Res}_{\phi}\left(\vec{D}_{0}\right)$, is a smooth form on $X$. Furthermore, the classical transgression formula

$$
\phi\left(D_{F}\right)-\phi\left(\vec{D}_{s}\right)=d T_{s}
$$

involving smooth differential forms on $X$, converges in the sense of currents as $s \rightarrow 0$, to the current equation

$$
\phi\left(D_{F}\right)-\phi\left(\left(\vec{D}_{0}\right)\right)=d T
$$

on $X$,

where the transgression current $T:=\lim _{s \rightarrow 0} T_{s}$ converges in $L_{\text {loc }}^{1}(X)$.

Let $\operatorname{Hom}(E, F) \rightarrow X$ denote the vector bundle of all bundle maps from $E$ to $F$. This bundle can be compactified in the vertical directions by embedding it in the bundle of projective spaces $\mathbb{P}(E \oplus F) \rightarrow X$. This embedding is defined by sending a bundle map to its graph. Recall that $\mathbb{P}(E \oplus F)=\operatorname{Hom}(E, F) \sqcup \mathbb{P}(F)$. Each section $\nu$ of $\mathbb{P}(E \oplus F) \rightarrow X$ induces a bundle map $E \stackrel{\alpha}{\rightarrow} F$ defined over the complement of the pole set $P:=\nu^{-1}(\mathbb{P}(F))$ of $\nu$ in $X$. We can apply the work of Harvey and Lawson described above to the induced map $\alpha$ over $X \sim P$, to construct the family $\left(\vec{D}_{s}, F\right)$ over $X \sim P$ and compute the Chern current at time zero on $X \sim P$. The main results of this paper can be stated as follows. The Chern forms, $\phi\left(\vec{D}_{s}\right)$ for $0<s<\infty$, extend smoothly across $P$ to all of $X$. Provided that the section $\nu$ of $\mathbb{P}(E \oplus F) \rightarrow X$ satisfies a suitable atomic hypothesis (see Definition 1.3), the Chern forms $\phi\left(\vec{D}_{s}\right)$ converge, in the sense of currents as $s \rightarrow \infty$, to the Chern current at time infinity, $\phi\left(\left(\vec{D}_{\infty}\right)\right)$, on $X$. Furthermore, the Chern current $\phi\left(\left(\vec{D}_{\infty}\right)\right)$ is of the form

$$
\phi\left(\left(\vec{D}_{\infty}\right)\right)=\phi\left(D_{F}\right)+\nu^{*}\left(\operatorname{Res}_{\phi}\left(\vec{D}_{\infty}\right)\right) \operatorname{Div}_{\infty}(\nu) \quad \text { on } X,
$$

where the pole divisor, $\operatorname{Div}_{\infty}(\nu)$, of $\nu$ is an integrally flat current supported on the pole set $P$ of $\nu$ and the residue form at infinity, $\operatorname{Res}_{\phi}\left(\vec{D}_{\infty}\right)$, is a $d$-closed smooth form on $\mathbb{P}(F)$ whose cohomology class can be computed in terms of the Chern classes of the bundles $E, F$ and of the tautological bundle $L$ over $\mathbb{P}(F)$. Further properties of the residue form at infinity will be discussed in the body of the paper.

In addition, the smooth transgression forms $T_{r, s}$, which satisfy the transgression formula

$$
\phi\left(\vec{D}_{r}\right)-\phi\left(\vec{D}_{s}\right)=d T_{r, s}
$$

for $0<s<r<\infty$ on $X \sim P$, extend smoothly to all of $X$. As $r \rightarrow \infty$ and $s \rightarrow 0$, the transgression forms $T_{r, s}$ converge in $L_{\text {loc }}^{1}(X)$ to the $L_{\text {loc }}^{1}$ transgression current $T$ and the current equation

$$
\phi\left(\left(\vec{D}_{\infty}\right)\right)-\phi\left(\left(\vec{D}_{0}\right)\right)=d T
$$


is the limiting form of the smooth transgression formula.

The main result outlined above is nicely illustrated by seeing what it says in the trivial case. Let $E=\underline{\mathbb{C}}$ and $F=\underline{\mathbb{C}}^{n}$ be the trivial rank 1 and $n$ bundles over $X=\mathbb{P}\left(\mathbb{C} \oplus \mathbb{C}^{n}\right)$, endowed with the trivial connection $d$. Let 0 denote the distinguished point $\mathbb{C} \oplus 0 \in \mathbb{P}\left(\mathbb{C} \oplus \mathbb{C}^{n}\right)$ and let $\mathbb{P}\left(\mathbb{C}^{n}\right)$ be the hyperplane at infinity in $\mathbb{P}\left(\mathbb{C} \oplus \mathbb{C}^{n}\right)$. The identity section $\nu$ of $\mathbb{P}\left(\underline{\mathbb{C}} \oplus \underline{\mathbb{C}}^{n}\right) \rightarrow \mathbb{P}\left(\mathbb{C} \oplus \mathbb{C}^{n}\right)$ is atomic with $\operatorname{Div}_{0}(\nu)=[0]$ and $\operatorname{Div}_{\infty}(\nu)=\left[\mathbb{P}\left(\mathbb{C}^{n}\right)\right]$, where $[M]$ denotes the current of integration over a submanifold $M$ of $X$. Then the $c_{n}$-Chern currents at time zero and time infinity on $\mathbb{P}\left(\mathbb{C} \oplus \mathbb{C}^{n}\right)$ are given by

$$
c_{n}\left(\left(\vec{D}_{0}\right)\right)=[0] \quad \text { and } \quad c_{n}\left(\left(\vec{D}_{\infty}\right)\right)=\left(-c_{1}\left(D_{L}\right)\right)^{n-1}\left[\mathbb{P}\left(\mathbb{C}^{n}\right)\right],
$$

where $D_{L}$ is the connection induced on the tautological line bundle $L \subset \underline{\mathbb{C}} \oplus \underline{\mathbb{C}}^{n}$ by the trivial connection $d$. Note that the residue at infinity, $\left(-c_{1}\left(D_{L}\right)\right)^{n-1}$, is just the volume form on $\mathbb{P}\left(\mathbb{C}^{n}\right)$ in the Fubini-Study metric. Finally, the smooth volume forms $c_{n}\left(D_{Q, s}\right)$ on $\mathbb{P}\left(\mathbb{C} \oplus \mathbb{C}^{n}\right)$ can be regarded as a compactification of a natural family of approximate identities on $\mathbb{C}^{n}$. This last fact can be generalized by saying that, in the universal case, the smooth forms $c_{n}\left(D_{Q, s}\right)$ on $\mathbb{P}(\underline{\mathbb{C}} \oplus F)$ compactify a smooth family of Thom forms on the bundle $F$.

The main ideas used to prove that the smooth Chern and transgression forms, $\phi\left(\vec{D}_{s}\right)$ and $T_{r, s}$ for $0<s<r<\infty$, on $X \sim P$ extend smoothly to all of $X$ can be outlined as follows. Let $\mathbf{E}, \mathbf{F}$ be the pullbacks to $\mathbb{P}(E \oplus F)$ of $E, F$, and let $U \subset \mathbf{E} \oplus \mathbf{F}$ be the tautological line bundle over $\mathbb{P}(E \oplus F)$. We begin by constructing a certain family of smooth connections, $D_{Q, s}$ for $0<s<\infty$, on the pullback to $X$ via $\nu$ of the quotient bundle $Q=\mathbf{E} \oplus \mathbf{F} / U$ over $\mathbb{P}(E \oplus F)$. Then we show that the family $\left(\vec{D}_{s}, F\right)$ is gauge equivalent to the family $\left(D_{Q, s}, \nu^{*}(Q)\right)$ over the subset $X \sim P$ of $X$. That is, the family $\left(\vec{D}_{s}, F\right)$, which is only defined over $X \sim P$, is compactified by the family $\left(D_{Q, s}, \nu^{*}(Q)\right)$, defined over all of $X$. Consequently the Chern and transgression forms of the family $\left(\vec{D}_{s}, F\right)$ over $X \sim P$ extend smoothly to all of $X$ as the Chern and transgression forms of the family $\left(D_{Q, s}, \nu^{*}(Q)\right)$ over $X$. In addition, we will show that as $s \rightarrow \infty$ the family $D_{Q, s}$ converges smoothly over the complement of the pole set $P$ of $\nu$ to a connection $D_{Q, \infty}$ defined on $\nu^{*}(Q)$ over $X \sim P$. Similarly, as the parameter $s \rightarrow 0$, the family $D_{Q, s}$ converges smoothly over the complement of the zero set $Z$ of $\nu$ in $X$ to a connection $D_{Q, 0}$ defined on $\nu^{*}(Q)$ over $X \sim Z$. This last fact implies that the Chern current at time zero on $X$ associated with an atomic section $\nu$ of $\mathbb{P}(E \oplus F) \rightarrow X$ has exactly the same form as it did in the case of a bundle map $\alpha$.

There are analogues of these results in the real case (see [HL, IV] and [Z2]) and in the case of $C^{\infty}$-meromorphic maps between quaternionic line bundles (see [HL, V] and Section 6). 


\section{Contents.}

1. Divisors of atomic sections of a compactified bundle . . . . . . 347

2. The induced singular connections and their smooth approximations . . . . . . . . . . . . . . . . . . . . . 349

3. The transgression and Chern currents . . . . . . . . . . 357

4. Residues at infinity . . . . . . . . . . . . . . 363

5. The relationship to Levine's construction . . . . . . . . . . . 370

6. Meromorphic quaternionic line bundle homomorphisms . . . . 376

Note that Sections 4, 5 and 6 can be read independently of each other.

1. Divisors of atomic sections of a compactified bundle. Let $E$ and $F$ be hermitian bundles of ranks 1 and $n$ over an oriented manifold $X$. In this section we apply the work of Harvey and Semmes [HS] to define the notion of an atomic section of the compactification $\mathbb{P}(E \oplus F) \rightarrow X$ of the bundle $\operatorname{Hom}(E, F) \rightarrow X$, define the zero and pole divisors of such a section and give some geometric conditions which ensure that a section is atomic.

We begin by recalling Harvey and Semmes definition of the divisor. (See [HS] for precise definitions.) The solid angle kernel, $\vartheta$, is the $L_{\text {loc }}^{1}$ form on $\mathbb{R}^{m}$ obtained by pulling back the volume form on the unit sphere to $\mathbb{R}^{m} \sim\{0\}$ by the radial projection map. The current equation $d\left(\gamma_{m}^{-1} \vartheta\right)=[0]$ on $\mathbb{R}^{m}$, where [0] denotes the point mass at the origin and $\gamma_{m}=\operatorname{Vol}\left(S^{m-1}\right)$, motivates the definition of divisor. Let $V \rightarrow X$ be a smooth real vector bundle of rank $m$ and let $M$ be a smooth manifold. A smooth map $f: M \rightarrow V$ is called atomic with respect to $X$ if for each choice of positively oriented local fibre coordinate $y$ on $V$ the smooth form $(y \circ f)^{*} \vartheta$ on $M \sim f^{-1}(X)$ has an $L_{\text {loc }}^{1}$ extension across $f^{-1}(X)$. Then the divisor of $f$ with respect to $X$, denoted $\operatorname{Div}_{X}(f)$, is defined by

$$
\operatorname{Div}_{X}(f):=d\left(\gamma_{m}^{-1}(y \circ f)^{*} \vartheta\right) \quad \text { on } M .
$$

Note that $\operatorname{Div}_{X}(f)$ is a degree $m$ current supported on the subset $f^{-1}(X)$ of $M$ and that the divisor of a section $f$ of $V \rightarrow X$ is simply the divisor of $f$ with respect to $X$.

Let $\mathbb{P}(E \oplus F) \rightarrow X$ denote the bundle of projective spaces whose fibre over a point $x \in X$ is the set of all one dimensional complex subspaces of $E_{x} \oplus F_{x}$. The ideas presented above can be applied to a section of the compactified bundle $\mathbb{P}(E \oplus F) \rightarrow X$ as follows. First we recall some of the basic structure of the bundle $\mathbb{P}(E \oplus F) \rightarrow X$. The natural embedding,

$$
\begin{aligned}
\operatorname{Hom}(E, F) & \hookrightarrow \mathbb{P}(E \oplus F), \\
\alpha_{x} & \mapsto \operatorname{Graph}\left(\alpha_{x}\right),
\end{aligned}
$$


where $\operatorname{Graph}\left(\alpha_{x}\right)=\left\{\left[v, \alpha_{x}(v)\right] \in \mathbb{P}\left(E_{x} \oplus F_{x}\right) / v \in E_{x} \sim\{0\}\right\}$, provides a compactification of the bundle $\operatorname{Hom}(E, F) \rightarrow X$ in the vertical directions. The total space of the projectivized bundle $\mathbb{P}(F) \rightarrow X$ can be regarded as a complexcodimension 1 submanifold of $\mathbb{P}(E \oplus F)$ by the inclusion

$$
\begin{aligned}
\mathbb{P}(F) & \hookrightarrow \mathbb{P}(E \oplus F), \\
{[f] } & \mapsto \quad[0, f] .
\end{aligned}
$$

Note that $\mathbb{P}(E \oplus F)=\operatorname{Hom}(E, F) \sqcup \mathbb{P}(F)$. Also recall that $X$ embeds in $\mathbb{P}(E \oplus$ $F)$ as the image of the zero section of $\operatorname{Hom}(E, F) \subset \mathbb{P}(E \oplus F)$ and that the projection

$$
\begin{aligned}
\pi: \mathbb{P}(E \oplus F) & \sim X \longrightarrow \mathbb{P}(F), \\
{[e, f] } & \longmapsto[f],
\end{aligned}
$$

has the natural structure of a complex line bundle. Finally, let $Z=\nu^{-1}(X)$ denote the zero set and $P=\nu^{-1}(\mathbb{P}(F))$ the pole set of a section $\nu$ of $\mathbb{P}(E \oplus F) \rightarrow$ $X$. Note that $Z \cap P=\varnothing$.

Definition 1.3. A smooth section $\nu: X \rightarrow \mathbb{P}(E \oplus F)$ is called atomic if the following two conditions hold.

(1) The induced section $\nu: X \sim P \rightarrow \operatorname{Hom}(E, F)$ is atomic.

(2) The induced mapping $\nu: X \sim Z \rightarrow \mathbb{P}(E \oplus F) \sim X$ is atomic with respect to $\mathbb{P}(F)$.

Thanks to [HS, 1.2] the zero and pole sets of an atomic section of the bundle $\mathbb{P}(E \oplus F) \rightarrow X$ have Lebesgue measure zero.

Definition 1.4. Let $\nu$ be an atomic section of the bundle $\mathbb{P}(E \oplus F) \rightarrow X$. Then:

(1) The zero divisor of $\nu$, denoted $\operatorname{Div}_{0}(\nu)$, is defined to be the extension from $X \sim P$ to all of $X$ of the divisor of the induced section $\nu: X \sim P \rightarrow$ $\operatorname{Hom}(E, F)$.

(2) The pole divisor of $\nu$, denoted $\operatorname{Div}_{\infty}(\nu)$, is defined to be the extension from $X \sim Z$ to all of $X$ of the divisor with respect to $\mathbb{P}(F)$ of the induced section $\nu: X \sim Z \rightarrow \mathbb{P}(E \oplus F) \sim X$.

Note that the zero (resp. pole) divisor of an atomic section of $\mathbb{P}(E \oplus F) \rightarrow X$ is a codimension (or degree) $2 n$ (resp. 2) $d$-closed flat current on $X$.

Remark 1.5. Harvey and Semmes prove that a large class of smooth sections of $\mathbb{P}(E \oplus F) \rightarrow X$ are atomic. More specifically, those sections of $\mathbb{P}(E \oplus F) \rightarrow X$ which approach their zero and pole sets algebraically and whose zero and pole sets are not too big in the sense of Minkowski are atomic. In addition, they show that the zero and pole divisors have some very beautiful structure. The reader is encouraged to consult [HS] for more information. 
2. The induced singular connections and their smooth approximations. We begin this section by recalling the definition of the singular pushforward and pullback connections and their smooth approximating families. Then we define the Chern currents at time zero and time infinity and review Harvey and Lawson's results concerning the Chern current at time zero. Next we present the main result of this section, proving that the smooth approximating Chern and transgression forms, defined by Harvey and Lawson on the complement of the pole set of a section $\nu$ of $\mathbb{P}(E \oplus F) \rightarrow X$, have a natural extension to all of $X$. Finally, we derive a formula for the smooth approximating transgression form in a local homogeneous coordinate system on $\mathbb{P}(E \oplus F)$. This formula will be useful in Section 3 where we address the problem of computing the Chern current at time infinity.

Let $E$ and $F$ be rank 1 and $\operatorname{rank} n$ hermitian bundles endowed with connections $D_{E}$ and $D_{F}$. The Chern forms of a connection $D$ on a complex vector bundle $V$ of rank $m$ are defined as usual (c.f. $[\mathrm{BC}]$ ). Let $\omega$ be a locally defined connection matrix or gauge for $D$ and let $\Omega:=d \omega-\omega \wedge \omega$ be the corresponding curvature matrix. The total Chern form $c$ is defined by

$$
c(D)=c(\Omega):=\operatorname{det}\left(I+\frac{i}{2 \pi} \Omega\right)
$$

and the Chern character is defined by

$$
\operatorname{ch}(D)=\operatorname{ch}(\Omega):=\operatorname{tr}\left(e^{i / 2 \pi \Omega}\right) .
$$

More generally, for any polynomial $\phi$ on $\mathfrak{g l}(m, \mathbb{C})$ which is invariant under the adjoint action, the $\phi$-Chern form is defined by

$$
\phi(D):=\phi(\Omega) .
$$

Let $D_{s}(0<s<\infty)$ be a family of connections on $V$. Set $\phi(A ; B):=$ $d /\left.d t\right|_{t=0} \phi(B+t A)$. The transgression form $T_{r, s}$ on $X$, which satisfies the standard transgression formula

$$
\phi\left(D_{r}\right)-\phi\left(D_{s}\right)=d T_{r, s} \quad \text { on } X,
$$

is defined (c.f. $[\mathrm{BC}])$ by

$$
T_{r, s}:=\int_{s}^{r} \phi\left(\frac{\partial \omega_{s}}{\partial s} ; \Omega_{s}\right) d s
$$

Following [HL] the connection $D_{E}$ on $E$ can be pushed forward via a section $\nu$ of $\mathbb{P}(E \oplus F) \rightarrow X$ to a singular connection $\vec{D}$ defined on $F$ over $X \sim(Z \cup P)$ as follows. 
Definition 2.3. Let $E \stackrel{\alpha}{\rightarrow} F$ over $X \sim P$ be the bundle map induced from a section $\nu$ of $\mathbb{P}(E \oplus F) \rightarrow X$ by the inclusion (1.1). The singular pushforward connection associated with $\nu$ is the smooth connection $\vec{D}$ on $F$ over $X \sim(Z \cup P)$ defined by

$$
\vec{D}:=\alpha D_{E} \beta+D_{F}(1-\alpha \beta)=D_{F}-\left(D_{F} \alpha-\alpha D_{E}\right) \beta,
$$

where $F \stackrel{\beta}{\rightarrow} E$ is the bundle map over $X \sim(Z \cup P)$ given by orthogonal projection of $F$ onto the subbundle $\operatorname{Im} \alpha$ followed by the inverse of the map $E \stackrel{\alpha}{\rightarrow}$ $\operatorname{Im} \alpha \subset F$.

Similarly, the connection $D_{F}$ on $F$ can be pulled back by $\nu$ to a connection $\overleftarrow{D}$ on $E$ over $X \sim(Z \cup P)$

Definition 2.4. The singular pullback connection associated with $\nu$ is the smooth connection $\overleftarrow{D}$ on $E$ over $X \sim(Z \cup P)$ defined by

$$
\overleftarrow{D}:=\beta D_{F} \alpha+(1-\beta \alpha) D_{E}=D_{E}+\beta\left(D_{F} \alpha-\alpha D_{E}\right)
$$

Note. Let $F \stackrel{\alpha^{*}}{\rightarrow} E$ denote the adjoint of $\alpha$. Then the bundle map $\beta$ defined above is given by

$$
\beta=\left(\alpha^{*} \alpha\right)^{-1} \alpha^{*}
$$

Harvey and Lawson considered the case of a smooth bundle map $E \stackrel{\alpha}{\rightarrow} F$ over $X$ (i.e., $P=\varnothing$ ). One of their main aims was to compute "Chern currents" for the singular connection $\vec{D}$ over $X$. To do this they constructed a smooth family $\vec{D}_{s}$, for $0<s<\infty$, of smooth connections on $F$ over all of $X$ so that as $s \rightarrow 0$, $\vec{D}_{s} \rightarrow \vec{D}$ as smooth connections on $F$ over $X \sim Z$, and as $s \rightarrow \infty, \vec{D}_{s} \rightarrow D_{F}$ as smooth connections on $F$ over $X$.

\section{Definition 2.5.}

(1) Such a family $\vec{D}_{s}$ is called a smooth approximating family to the singular connection $\vec{D}$.

(2) If as $s \rightarrow 0$ the corresponding Chern forms $\phi\left(\vec{D}_{s}\right)$ on $X$ converge weakly as currents on $X$, then the limit will be denoted $\phi\left(\left(\vec{D}_{0}\right)\right)$ and referred to as the $\phi$-Chern current of the singular connection $\vec{D}$.

Harvey and Lawson use an "approximate one" (c.f. [HL I.4.1]) to construct a smooth approximating family to the singular connection $\vec{D}$. Different choices of approximate one yield different approximating families. Because of its geometrical significance (see Theorems 2.19 and 2.21 below) we will restrict our attention to the algebraic approximation mode $\chi:[0, \infty] \rightarrow[0,1]$ defined by $\chi(t)=t /(1+t)$.

Returning to the case of a section $\nu$ of $\mathbb{P}(E \oplus F) \rightarrow X$ we make the following definitions. 
Definition 2.6. The singular pushforward connection $\vec{D}$ associated with $\nu$ is smoothed over $X \sim P$ by the approximating family of smooth connections $\vec{D}_{s}(0<s \leq \infty)$ on $F$ over $X \sim P$ defined by

$$
\vec{D}_{s}=D_{F}+\left(\alpha D_{E}-D_{F} \alpha\right) \alpha^{*}\left(\alpha \alpha^{*}+s^{2}\right)^{-1}=\left(s^{2} D_{F}+\alpha D_{E} \alpha^{*}\right)\left(\alpha \alpha^{*}+s^{2}\right)^{-1} \text {. }
$$

Note that as $s \rightarrow 0, \vec{D}_{s} \rightarrow \vec{D}_{0}=\vec{D}$ as smooth connections on $F$ over $X \sim(Z \cup P)$, and as $s \rightarrow \infty, \vec{D}_{s} \rightarrow D_{F}$ as smooth connections on $F$ over $X \sim P$.

Similarly, in the pullback case we define

$$
\begin{aligned}
\overleftarrow{D}_{s} & :=D_{E}+\left(\alpha^{*} \alpha+s^{2}\right)^{-1} \alpha^{*}\left(D_{F} \alpha-\alpha D_{E}\right) \\
& =\left(\alpha^{*} \alpha+s^{2}\right)^{-1}\left(s^{2} D_{E}+\alpha^{*} D_{F} \alpha\right) .
\end{aligned}
$$

Note that $\overleftarrow{D}_{\infty}=D_{E}$

To simplify the discussion we will restrict our attention to the pushforward case. The discussion in the pullback case proceeds along similar lines. We will show that for $0<s<\infty$, the Chern forms $\phi\left(\vec{D}_{s}\right)$ and the corresponding transgression forms $T_{r, s}$ defined initially over $X \sim P$ extend across the pole set $P$ to smooth forms on all of $X$. Suppose now that $\nu$ is an atomic section of $\mathbb{P}(E \oplus F) \rightarrow X$. Harvey and Lawson showed that the Chern current at time zero,

$$
\phi\left(\left(\vec{D}_{0}\right)\right):=\lim _{s \rightarrow 0} \phi\left(\vec{D}_{s}\right)
$$

exists (see Theorem 2.25). The main aim of this paper is to show that the Chern current at time infinity,

$$
\phi\left(\left(\vec{D}_{\infty}\right)\right):=\lim _{r \rightarrow \infty} \phi\left(\vec{D}_{r}\right)
$$

exists on all of $X$, and to compute it.

Now, the families $\left(\vec{D}_{s}, F\right)$ and $\left(\overleftarrow{D}_{s}, E\right)$ for $0<s<\infty$ do not extend from $X \sim P$ to all of $X$. However, we will construct families $\left(D_{Q, s}, Q\right)$ and $\left(D_{U, s}, U\right)$ defined over all of $X$, whose Chern and transgression forms are smooth extensions to all of $X$ of the Chern and transgression forms of the families $\left(\vec{D}_{s}, F\right)$ and $\left(\overleftarrow{D}_{s}, E\right)$ on $X \sim P$. The construction of the families $\left(D_{Q, s}, Q\right)$ and $\left(D_{U, s}, U\right)$ which relies heavily on [HL, I.8], is most easily described in the universal case. 
Remark 2.12. The universal case. Let $\mathbf{E}=p^{*} E$ and $\mathbf{F}=p^{*} F$ be the pullbacks of $E$ and $F$ to $\mathbb{P}(E \oplus F)$ via the projection map $p: \mathbb{P}(E \oplus F) \rightarrow X$, and let $\mathbf{D}_{\mathbf{E}}$ and $\mathbf{D}_{\mathbf{F}}$ be the pullbacks to $\mathbf{E}$ and $\mathbf{F}$ of the connections $D_{E}$ and $D_{F}$. The induced bundle $\mathbb{P}(\mathbf{E} \oplus \mathbf{F}) \rightarrow \mathbb{P}(E \oplus F)$ has a tautological or universal section $\boldsymbol{\nu}$ which sends a line $\ell \in \mathbb{P}(E \oplus F)$ to itself. The section $\boldsymbol{\nu}$ is atomic with zero divisor $\operatorname{Div}_{0}(\boldsymbol{\nu})=[X]$ and pole divisor $\operatorname{Div}_{\infty}(\boldsymbol{\nu})=[\mathbb{P}(F)]$. For any section $\nu$ of $p: \mathbb{P}(E \oplus F) \rightarrow X$, we have $\nu^{*} \mathbf{E}=E$ and $\nu^{*} \mathbf{F}=F$ as bundles with connections, and $\nu^{*}(\boldsymbol{\nu})=\nu$. So to prove results of a smooth nature on or over $X$ which involve a section $\nu$, it often suffices to prove them on or over $\mathbb{P}(E \oplus F)$ for the universal section $\boldsymbol{\nu}$ and then pullback the result to $X$ via $\nu$.

Definition 2.13. Let $V, W$ be isomorphic vector bundles. A family of connections $D_{V, s}$ on $V$ is said to be equivalent to a family of connections $D_{W, s}$ on $W(0<s<\infty)$ if there is a bundle isomorphism $\varphi: V \rightarrow W$ (independent of $s$ ) so that

$$
D_{W, s}=\varphi \circ D_{V, s} \circ \varphi^{-1}
$$

for all $s \in(0, \infty)$.

This concept is important because of the following fact.

Lemma 2.15. Equivalent families of connections have equal Chern and transgression forms.

In the universal case the family $\left(D_{U, s}, U\right)$ is constructed over $\mathbb{P}(E \oplus F)$ as follows. Let $U$ denote the tautological line bundle over $\mathbb{P}(E \oplus F)$ and let $U_{s}$ $(0<s<\infty)$ be the pullback of $U$ over $\mathbb{P}(E \oplus F)$ by the flow

$$
\begin{aligned}
\Psi_{s}: \mathbb{P}(E \oplus F) & \longrightarrow \mathbb{P}(E \oplus F), \\
{[e, f] } & \longmapsto[s e, f] .
\end{aligned}
$$

We can induce a connection $D_{U_{s}}$ on $U_{s}$ over $\mathbb{P}(E \oplus F)$ by regarding $U_{s}$ as a subbundle of $\mathbf{E} \oplus \mathbf{F}$ and defining

$$
D_{U_{s}}=\operatorname{pr}_{s} \circ \mathbf{D}_{\mathbf{E} \oplus \mathbf{F}} \quad \text { operating on sections of } U_{s},
$$

where $\operatorname{pr}_{s}: \mathbf{E} \oplus \mathbf{F} \rightarrow U_{s}$ denotes orthogonal projection. Then we define the family of connections $D_{U, s}$ on $U$ over $\mathbb{P}(E \oplus F)$ by

$$
D_{U, s}:=\Psi_{s}^{-1} \circ D_{U_{s}} \circ \Psi_{s},
$$

where we now regard $\Psi_{s}$ as a bundle map from $U$ to $U_{s}$.

The following result is a corollary of Theorem I.8.14 of [HL]. Note that the polynomials $c_{1}, c_{1}^{2}, c_{1}^{3}, \ldots$ generate the set of all Ad-invariant polynomials on $\mathfrak{g l}(1, \mathbb{C})$ and that $c^{-1}:=\left(1+c_{1}\right)^{-1}=\sum_{j=0}^{\infty}(-1)^{j} c_{1}^{j}$. 


\section{Theorem 2.19.}

(1) The family $\left(\overleftarrow{D}_{s}, \mathbf{E}\right)(0<s<\infty)$ is equivalent over $\operatorname{Hom}(E, F)$ to the family $\left(D_{U, s}, U\right)$. More specifically,

$$
D_{U, s}=\gamma \circ \overleftarrow{D}_{s} \circ \gamma^{-1}
$$

over $\operatorname{Hom}(E, F)$

where $\gamma: \mathbf{E} \rightarrow U$ is the graphing map, $\gamma(e):=(e, \alpha(e))$, for $e \in \mathbf{E}_{\alpha}$.

(2) Therefore, the Chern forms $c^{-1}\left(\overleftarrow{D}_{s}\right)(0<s<\infty)$ and the transgression forms $T_{r, s}(0<s<r<\infty)$ of the family $\left(\overleftarrow{D}_{s}, E\right)$ on $\operatorname{Hom}(E, F)$ extend smoothly to all of $\mathbb{P}(E \oplus F)$ as the Chern and transgression forms of the family $\left(D_{U, s}, U\right)$ over $\mathbb{P}(E \oplus F)$.

The corresponding result in the pushforward case can be described as follows. Let $G_{n}\left(F^{*} \oplus E^{*}\right)$ denote the Grassmannian of $n$-planes in $F^{*} \oplus E^{*}$, and let $U_{n}$ be the tautological $\operatorname{rank} n$ bundle over $G_{n}\left(F^{*} \oplus E^{*}\right)$. Recall that there is a natural bundle isomorphism,

$$
\varphi: \mathbb{P}(E \oplus F) \rightarrow G_{n}\left(F^{*} \oplus E^{*}\right),
$$

defined by sending a line to its annihilator, and that the pullback $\varphi^{*}\left(U_{n}\right)$ to $\mathbb{P}(E \oplus F)$ of the tautological bundle over $G_{n}\left(F^{*} \oplus E^{*}\right)$ is the dual $Q^{*}$ of the quotient bundle $Q=\mathbf{E} \oplus \mathbf{F} / U$. Let $D_{U_{n}, s}$ denote the connection defined on $U_{n} \rightarrow G_{n}\left(F^{*} \oplus E^{*}\right)$ as in (2.18).

Definition 2.20. The family of connections $D_{Q, s}$ on $Q$ over $\mathbb{P}(E \oplus F)$ is defined to be the family dual to the family of connections induced on $\varphi^{*}\left(U_{n}\right)$ by the family $D_{U_{n}, s}$ on $U_{n}$ over $G_{n}\left(F^{*} \oplus E^{*}\right)$.

\section{Theorem 2.21.}

(1) The family $\left(\vec{D}_{s}, \mathbf{F}\right)(0<s<\infty)$ is equivalent over $\operatorname{Hom}(E, F)$ to the family $\left(D_{Q, s}, Q\right)$. More specifically,

$$
D_{Q, s}=\psi \circ \vec{D}_{s} \circ \psi^{-1} \quad \text { over } \operatorname{Hom}(E, F),
$$

where the bundle isomorphism $\psi: \mathbf{F} \rightarrow Q$ over $\operatorname{Hom}(E, F)$ is defined by

$$
\psi(f):=[0, f] \quad \text { for } f \in \mathbf{F} .
$$

Here $[e, f]$ denotes the image of $(e, f)$ in $Q$.

(2) Therefore, the Chern forms $\phi\left(\vec{D}_{s}\right)(0<s<\infty)$ and the transgression forms $T_{r, s}(0<s<r<\infty)$ of the family $\left(\vec{D}_{s}, F\right)$ on $\operatorname{Hom}(E, F)$ extend smoothly to all of $\mathbb{P}(E \oplus F)$ as the Chern and transgression forms of the family $\left(D_{Q, s}, Q\right)$ over $\mathbb{P}(E \oplus F)$. 
Note. As suggested in Remark 2.12, Theorems 2.19 and 2.21 also hold for a smooth section $\nu$ of $\mathbb{P}(E \oplus F) \rightarrow X$ provided that we replace all spaces, bundles, connections and bundle maps by their pullbacks to $X$ via $\nu$.

Proof. First note that the restriction of the mapping $\varphi$ to the finite part $\operatorname{Hom}(E, F)$ of $\mathbb{P}(E \oplus F)$ is given by

$$
\begin{aligned}
\operatorname{Hom}(E, F) & \stackrel{\varphi}{\longrightarrow} \operatorname{Hom}\left(F^{*}, E^{*}\right), \\
\alpha & \longmapsto-\tilde{\alpha},
\end{aligned}
$$

where the dual homomorphism $\tilde{\alpha}$ is defined by $\tilde{\alpha}\left(f^{*}\right)(e):=f^{*}(\alpha(e))$. Now by Theorem 2.19 , the family $\left(\overleftarrow{D}_{s, F^{*}}, \mathbf{F}^{*}\right)$ is equivalent over $\operatorname{Hom}\left(F^{*}, E^{*}\right)$ to the family $\left(D_{U_{n}, s}, U_{n}\right)$. The isomorphism $\tilde{\gamma}: \mathbf{F}^{*} \rightarrow U_{n}$ setting up this equivalence is given by $\tilde{\gamma}\left(f^{*}\right)=\left(f^{*}, \tilde{\alpha}\left(f^{*}\right)\right)$. This data pulls back via the mapping $\varphi$ : $\operatorname{Hom}(E, F) \rightarrow \operatorname{Hom}\left(F^{*}, E^{*}\right)$ of $(2.22)$ to the isomorphism

$$
\left(\mathbf{F}^{*}, \overleftarrow{D}_{s, F^{*}}\right) \stackrel{\varphi^{*}(\tilde{\gamma})}{\longrightarrow}\left(\varphi^{*}\left(U_{n}\right), \varphi^{*}\left(D_{U_{n}, s}\right)\right) \quad \text { over } \operatorname{Hom}(E, F)
$$

Since $\vec{D}_{s, F}=\left(\overleftarrow{D}_{s, F^{*}}\right)^{*}$ on $\mathbf{F}$ (see [HL, I.4.28]) and

$$
\left(Q, D_{Q, s}\right)=\left(\varphi^{*}\left(U_{n}\right)^{*},\left(\varphi^{*}\left(D_{U_{n}, s}\right)\right)^{*}\right)
$$

the dual isomorphism is given by

$$
\left(Q, D_{Q, s}\right) \stackrel{\eta}{\longrightarrow}\left(\mathbf{F}, \vec{D}_{s}\right) \quad \text { over } \operatorname{Hom}(E, F),
$$

where $\eta:=\widetilde{\varphi^{*}(\tilde{\gamma})}$.

To complete the proof we just need to identify the map $\eta$ and show that its inverse is the mapping $\psi$. Let $f^{*} \in \mathbf{F}^{*}$ and $(e, f) \in \mathbf{E} \oplus \mathbf{F}$ be arbitrary. An element $[e, f] \in Q$ defines a linear functional $T_{[e, f]}$ on $\varphi^{*}\left(U_{n}\right)$ by $T_{[e, f]}\left(v^{*}, w^{*}\right)=$ $v^{*}(f)-w^{*}(e)$ for $\left(v^{*}, w^{*}\right) \in \varphi^{*}\left(U_{n}\right) \subset \mathbf{F}^{*} \oplus \mathbf{E}^{*}$. So by the definition of the dual map and using the identification of $\mathbf{F}$ with $\mathbf{F}^{* *}$ we have $\eta([e, f])\left(f^{*}\right)=$ $T_{[e, f]}\left(\tilde{\gamma}\left(f^{*}\right)\right)=f^{*}(f)-\tilde{\alpha}\left(f^{*}\right)(e)=(f-\alpha(e))\left(f^{*}\right)$ and so

$$
\eta([e, f])=f-\alpha(e) .
$$

Finally, it is easy to check that $\eta^{-1}(f)=[0, f]=\psi(f)$.

Note. The extensions of the Chern forms $c^{-1}\left(\overleftarrow{D}_{s}\right)$ and $\phi\left(\vec{D}_{s}\right)$ and the transgression forms $T_{r, s}$ from $\operatorname{Hom}(E, F)$ to all of $\mathbb{P}(E \oplus F)$ will also be denoted by $c^{-1}\left(\overleftarrow{D}_{s}\right), \phi\left(\vec{D}_{s}\right)$ and $T_{r, s}$ 
Lemma 2.23 ([HL, I.9.2]).

(1) Over $\mathbb{P}(E \oplus F) \sim X$ the smooth family of subbundles $U_{s} \subset \mathbf{E} \oplus \mathbf{F}$ for $0<$ $s<\infty$ extends smoothly to $s=0$, where $U_{0}=\pi^{*} L$ is the pullback to $\mathbb{P}(E \oplus F) \sim X$ of the universal bundle $L \rightarrow \mathbb{P}(F)$ via the natural map $\pi: \mathbb{P}(E \oplus F) \sim X \rightarrow \mathbb{P}(F)$ defined by (1.2).

(2) Over $\operatorname{Hom}(E, F) \subset \mathbb{P}(E \oplus F)$ the smooth family of subbundles $U_{s} \subset \mathbf{E} \oplus \mathbf{F}$ for $0<s<\infty$ extends smoothly to $s=\infty$ where $U_{\infty}=\mathbf{E}$.

Note.

(1) The bundle $U_{0}$ on $\mathbb{P}(E \oplus F) \sim X$ does not extend smoothly across $X$.

(2) Even though the bundle $U_{\infty}=\mathbf{E}$ on $\operatorname{Hom}(E, F)$ extends smoothly across $\mathbb{P}(F)$, the smooth family $U_{s}$ over $\operatorname{Hom}(E, F)$ for $0<s \leq \infty$ does not extend smoothly across $\mathbb{P}(F)$.

\section{Corollary 2.24.}

(1) The families $\left(D_{U, s}, U\right)$ and $\left(D_{Q, s}, Q\right)$ extend smoothly to $s=0$ over $\mathbb{P}(E \oplus F) \sim X$.

(2) The families $\left(D_{U, s}, U\right)$ and $\left(D_{Q, s}, Q\right)$ extend smoothly to $s=\infty$ over $\mathbb{P}(E \oplus F) \sim \mathbb{P}(F)$.

Combining this corollary with [HL, III.6.11] we have:

Theorem 2.25 ([HL, III.6.11]). Let $\nu$ be an atomic section of $\mathbb{P}(E \oplus F) \rightarrow$ $X$.

(1) The smooth form $\phi\left(\vec{D}_{0}\right)$ on $X \sim Z$ extends across $Z$ to a d-closed $L_{\text {loc }}^{1}$ form on all of $X$, also denoted by $\phi\left(\vec{D}_{0}\right)$.

(2) The Chern current $\phi\left(\left(\vec{D}_{0}\right)\right)$ exists on $X$ and equals

$$
\phi\left(\left(\vec{D}_{0}\right)\right)=\phi\left(\vec{D}_{0}\right)+\operatorname{Res}_{\phi}\left(\overleftarrow{D}_{0}\right) \operatorname{Div}_{0}(\nu)
$$

where the residue form, $\operatorname{Res}_{\phi}\left(\vec{D}_{0}\right)$, is a d-closed smooth form on $X$.

Corollary 2.24 also enables us to show that the Chern current $\phi\left(\left(\vec{D}_{\infty}\right)\right)$ exists on $X \sim P$ and equals $\phi\left(D_{F}\right)$. The next section is devoted to computing $\phi\left(\left(\vec{D}_{\infty}\right)\right)$ on all of $X$. In order to do this we need to find a local formula for the transgression form $T_{r, s}$ associated to a section $\nu$ of $\mathbb{P}(E \oplus F) \rightarrow X$. To describe this we work universally in a local homogeneous coordinate on $\mathbb{P}(E \oplus F)$ and pullback the resulting local formula to $X$ via $\nu$.

Harvey and Lawson derived the following local formula for the transgression form $T_{r, s}$. Let $\boldsymbol{\alpha}: \mathbf{E} \rightarrow \mathbf{F}$ be the tautological homomorphism over $\operatorname{Hom}(E, F)$. 
Let $e, f$ be local frames for $\mathbf{E}, \mathbf{F}$ and define a local fibre variable $u=\left(u_{1}, \ldots, u_{n}\right)$ on $\operatorname{Hom}(E, F)$ by $\boldsymbol{\alpha} e=u f$. Let $\boldsymbol{\alpha}^{*} f=u^{*} e$ define $u^{*}$. Set $|u|^{2}=u u^{*}$,

$$
D u=d u+u \omega_{F}-\omega_{E} u \text { and } D u^{*}=d u^{*}+u^{*} \omega_{E}-\omega_{F} u^{*} .
$$

Finally let $\partial / \partial u$ and $\partial / \partial u^{*}$ be the globally defined Euler and co-Euler vector fields on $\operatorname{Hom}(E, F)$ (c.f. [HL, I.5.24]). Then, in the pushforward case, we have ([HL, III.3.17]) that

$$
\begin{aligned}
T_{r, s} & =\int_{\chi_{r}}^{\chi_{s}} \phi\left(\frac{u^{*} D u}{|u|^{2}} ; \Lambda(x)\right) d x \\
& =-\int_{\chi_{r}}^{\chi_{s}} u^{*} \frac{\partial}{\partial u^{*}}\left\llcorner\phi(\Lambda(x)) \frac{d x}{x},\right.
\end{aligned}
$$

where $\chi_{s}=|u|^{2} /\left(s^{2}+|u|^{2}\right)$ and

$$
\Lambda(x)=\Omega_{F}+x\left(\frac{u^{*} \Omega_{E} u}{|u|^{2}}-\frac{u^{*} u}{|u|^{2}} \Omega_{F}-\frac{D u^{*} D u}{|u|^{2}}\right) .
$$

The formula for the transgression form $T_{r, s}$ in a local homogeneous coordinate on $\mathbb{P}(E \oplus F)$ is obtained as follows. Let $u$ be a local fibre variable on $\operatorname{Hom}(E, F)$ defined as above. Let $(t, u)$ be the local fibre variables on $E \oplus F$ defined by $c=(t e, u f)$ where $c: E \oplus F \rightarrow \mathbf{E} \oplus \mathbf{F}$ is the tautological section. We call $[t, u]$ the local homogeneous coordinate on $\mathbb{P}(E \oplus F)$ corresponding to the fibre variable $u$.

Let $\omega$ be a smooth form on $\mathbb{P}(E \oplus F)$. Suppose that over the subset $\operatorname{Hom}(E, F), \omega$ is expressed in terms of a local fibre variable $u$. Let $\omega_{H C}:=\pi^{*} \omega$ be the pullback of $\omega$ to $E \oplus F \sim X$ via the natural projection map $\pi: E \oplus F \sim$ $X \rightarrow \mathbb{P}(E \oplus F)$. Then to obtain an expression for $\omega_{H C}$ in the corresponding local homogeneous coordinate $(t, u)$ we simply replace $u$ by $u / t$ in the expression for $\omega$. The resulting expression for $\omega_{H C}$ will be called the expression for $\omega$ in homogeneous coordinates. The following general lemma will be of use in finding expressions for the transgression and Chern forms in homogeneous coordinates.

Lemma 2.29. Let $u$ be a local fibre coordinate on $\operatorname{Hom}(E, F)$. Let

$$
\mathcal{C}=\left\{\frac{u^{*} u}{|u|^{2}}, \frac{u^{*} D u}{|u|^{2}}, \frac{D u^{*} u}{|u|^{2}}, \frac{D u^{*} D u}{|u|^{2}}\right\} \cup \Omega^{*}(X),
$$

and set $\chi_{s}=|u|^{2} /\left(s^{2}+|u|^{2}\right)$. Let $\omega$ be a (possibly $\left.1 \times 1\right)$ matrix valued form on the total space of the bundle $\operatorname{Hom}(E, F) \rightarrow X$. Suppose that $\omega$ can be expressed as a polynomial in $\chi_{r}, \chi_{s}$ and $\partial \chi_{s} / \partial s$ with coefficients which are themselves 
polynomial expressions in (the entries of) the forms belonging to the set $\mathcal{C}$. Then the expression for $\omega$ in homogeneous coordinates $[t, u]$ on $\mathbb{P}(E \oplus F)$ is

$$
\begin{aligned}
\omega_{H C}=\omega-\frac{d t}{t}\left(u \frac{\partial}{\partial u}\llcorner\omega)\right. & -\frac{d \bar{t}}{\bar{t}}\left(u^{*} \frac{\partial}{\partial u^{*}}\llcorner\omega)\right. \\
& -\frac{d t d \bar{t}}{|t|^{2}}\left(u \frac { \partial } { \partial u } \left\llcorneru^{*} \frac{\partial}{\partial u^{*}}\llcorner\omega)\right.\right.
\end{aligned}
$$

where any occurrence of $\chi_{s}$ on the R.H.S. is replaced by $|u|^{2} /\left(s^{2}|t|^{2}+|u|^{2}\right)$.

Proof. First note that (2.30) holds for each $\omega \in \mathcal{C}$. For instance,

$$
\begin{aligned}
\left(\frac{D u^{*} D u}{|u|^{2}}\right)_{H C} & =\frac{\left(D u^{*}-u^{*} \frac{d \bar{t}}{\bar{t}}\right)\left(D u-u \frac{d t}{t}\right)}{|u|^{2}} \\
& =\frac{D u^{*} D u}{|u|^{2}}+\frac{d t}{t} \frac{D u^{*} u}{|u|^{2}}-\frac{d \bar{t}}{\bar{t}} \frac{u^{*} D u}{|u|^{2}}-\frac{d t d \bar{t}}{|t|^{2}} \frac{u^{*} u}{|u|^{2}}
\end{aligned}
$$

which has the required form. The proof is completed by checking that if $\omega$ and $\eta$ satisfy (2.30), then so do $\omega+\eta$ and $\omega \wedge \eta$.

Corollary 2.31. Let $T_{r, s}$ be the smooth transgression form on $\mathbb{P}(E \oplus F)$ associated to the family of Chern forms $\phi\left(\vec{D}_{s}\right)$. Then the expression for $T_{r, s}$ in a local homogeneous coordinate system $[t, u]$ on $\mathbb{P}(E \oplus F)$ is given by

$$
\left(T_{r, s}\right)_{H C}=T_{r, s}-\frac{d t}{t}\left(u \frac{\partial}{\partial u}\left\llcorner T_{r, s}\right)\right.
$$

where $T_{r, s}$ is defined by replacing $\chi_{s}$ by $|u|^{2} /\left(s^{2}|t|^{2}+|u|^{2}\right)$ in (2.26) or (2.27).

3. The transgression and Chern currents. In this section we compute the Chern currents at time infinity,

$$
\phi\left(\left(\vec{D}_{\infty}\right)\right):=\lim _{r \rightarrow \infty} \phi\left(\vec{D}_{r}\right) \quad \text { and } \quad c^{-1}\left(\left(\overleftarrow{D}_{\infty}\right)\right):=\lim _{r \rightarrow \infty} c^{-1}\left(\overleftarrow{D}_{r}\right)
$$

on $X$ associated with an atomic section $\nu$ of the bundle $\mathbb{P}(E \oplus F) \rightarrow X$. We begin with a discussion of the pushforward case. Let $T_{r, s}$ denote the transgression form induced on $X$ in the pushforward case by a section $\nu$ of $\mathbb{P}(E \oplus F) \rightarrow X$ and an invariant polynomial $\phi$. 
Proposition 3.1. Let $\nu$ be an atomic section of the bundle $\mathbb{P}(E \oplus F) \rightarrow X$. Then the transgression current $T_{\infty, 0}=\lim _{\substack{r \rightarrow \infty \\ s \rightarrow 0}} T_{r, s}$ converges in $L_{\text {loc }}^{1}(X)$ and equals

$$
\left(T_{\infty, 0}\right)_{H C}=T-\frac{d t}{t}\left(u \frac{\partial}{\partial u}\llcorner T)\right.
$$

in local homogeneous coordinates $[t, u]$ where

$$
T=\int_{0}^{1} \phi\left(\frac{u^{*} D u}{|u|^{2}} ; \Lambda(x)\right) d x .
$$

Here $\Lambda(x)$ is given by (2.28).

Proof. Since $\nu$ is atomic, $Z \cup P$ has Lebesgue measure zero in $X$ and so by Corollary 2.24, $T_{r, s} \rightarrow T_{\infty, 0}$ a.e. on $X$ as $r \rightarrow \infty$ and $s \rightarrow 0$. So to prove that $T_{r, s} \rightarrow T_{\infty, 0}$ in $L_{\text {loc }}^{1}(X)$, we just need to show that the Lebesgue Dominated Convergence Theorem applies. We work locally in the pullback via $\nu$ of the various charts on $\mathbb{P}(E \oplus F)$. Let $[t, u]$ be a local coordinate expression for $\nu$, defined with respect to a local frame $e, f$ for $E \oplus F$ by $\nu=[t e, u f]$. Recall that the coordinate expressions for the transgression form $T_{r, s}$ in the charts $\nu^{-1}(\mathcal{H})=\{t \neq 0\}$ and $\nu^{-1}\left(\mathcal{U}_{j}\right)=\left\{u_{j} \neq 0\right\}$ for $j \in\{1, \ldots, n\}$ are given by setting $t=1$ and $u_{j}=1$ for $j \in\{1, \ldots, n\}$, respectively, in (2.32). Also note that since $\nu$ is atomic, the local functions $t$ on $\nu^{-1}\left(\mathcal{U}_{j}\right)$ and $u$ on $\nu^{-1}(\mathcal{H})$ are atomic.

Using the atomicity of $u$ Harvey and Lawson [HL, III.2.8] have shown that $T_{r, s}$ converges to $T_{\infty, 0}$ in $L_{\text {loc }}^{1}\left(\nu^{-1}(\mathcal{H})\right)$. Consulting $(2.32)$ we see that in each of the charts $\nu^{-1}\left(\mathcal{U}_{j}\right)$, the transgression $T_{r, s}$ is a polynomial in $\chi_{r}$ and $\chi_{s}$ with coefficients which are either smooth forms on $\nu^{-1}\left(\mathcal{U}_{j}\right)$ or products of a smooth form on $\nu^{-1}\left(\mathcal{U}_{j}\right)$ with the $L_{\text {loc }}^{1}\left(\nu^{-1}\left(\mathcal{U}_{j}\right)\right)$ form $d t / t$. So, since $\chi_{r}$ and $\chi_{s}$ are bounded and converge to 0 and 1 a.e. as $r \rightarrow \infty$ and $s \rightarrow 0$, the atomicity of $t$ together with the Lebesgue Dominated Convergence Theorem imply that $T_{r, s} \rightarrow T_{\infty, 0}$ in $L_{\text {loc }}^{1}\left(\nu^{-1}\left(\mathcal{U}_{j}\right)\right)$. Patching these results together, we conclude that $T_{r, s}$ converges to $T_{\infty, 0}$ in $L_{\mathrm{loc}}^{1}(X)$.

Corollary 3.4. Let $\nu$ be an atomic section of the bundle $\mathbb{P}(E \oplus F) \rightarrow X$. Then the Chern current at time infinity, $\phi\left(\left(\vec{D}_{\infty}\right)\right):=\lim _{r \rightarrow \infty} \phi\left(\vec{D}_{r}\right)$, exists on $X$ and is of the form

$$
\phi\left(\left(\vec{D}_{\infty}\right)\right)=\phi\left(D_{F}\right)+S
$$

where $S$ is a flat current supported on the pole set $P$ of $\nu$. Furthermore, the current equation

$$
d T_{\infty, 0}=\phi\left(\left(\vec{D}_{\infty}\right)\right)-\phi\left(\left(\vec{D}_{0}\right)\right)
$$


is the current limit, as $s \rightarrow 0$ and $r \rightarrow \infty$, of the smooth transgression formula

$$
d T_{r, s}=\phi\left(\vec{D}_{r}\right)-\phi\left(\vec{D}_{s}\right) \quad \text { on } X .
$$

Proof. By Corollary 2.24(1) and Proposition 3.1, the Chern current

$$
\phi\left(\left(\vec{D}_{\infty}\right)\right):=\lim _{r \rightarrow \infty} \phi\left(\vec{D}_{r}\right)=\phi\left(\vec{D}_{0}\right)+d T_{\infty, 0}
$$

exists and is flat on $X \sim Z$, and hence on all of $X$ by Corollary 2.24(2). Finally, by $(2.8)$, the difference $S:=\phi\left(\left(\vec{D}_{\infty}\right)\right)-\phi\left(D_{F}\right)$ is a flat current supported on $P$.

The current $S$ is called the singular part of the Chern current, and has several nice properties which we now describe. First, we define the residue form at infinity, $\operatorname{Res}_{\phi}\left(\vec{D}_{\infty}\right)$. We work universally on $\mathbb{P}(E \oplus F)$. Set $\mathcal{R}:=\mathbb{P}(E \oplus$ $F) \sim X$. Choose a hermitian metric on the line bundle $\pi: \mathcal{R} \rightarrow \mathbb{P}(F)$ and let $\rho: S_{\varepsilon}(\mathcal{R}) \rightarrow \mathbb{P}(F)$ denote the $\varepsilon$-circle bundle in $\mathcal{R}$. Note that the restriction of the transgression current $T_{\infty, 0}$ to $S_{\varepsilon}(\mathcal{R})$ is a smooth form. Finally, suppose that the invariant polynomial $\phi$ has pure degree $m$.

Definition 3.5. The residue form at infinity is the smooth $2(m-1)$-form on $\mathbb{P}(F)$ defined by

$$
\operatorname{Res}_{\phi}\left(\vec{D}_{\infty}\right):=\int_{\rho^{-1}} T_{\infty, 0}=\rho_{*}\left(T_{\infty, 0}\right)
$$

Note that the residue form pulls back via the projection map $\pi$ to a smooth form on $\mathbb{P}(E \oplus F) \sim X$, also denoted by $\operatorname{Res}_{\phi}\left(\vec{D}_{\infty}\right)$.

Note. Similarly Harvey and Lawson define the residue form $\operatorname{Res}_{\phi}\left(\vec{D}_{0}\right)$ by integrating the transgression current $-T_{\infty, 0}$ over any $\varepsilon$-sphere bundle about $X$ in $\operatorname{Hom}(E, F) \rightarrow X$.

\section{Lemma 3.6.}

(1) The expression for the residue at infinity in a local homogeneous coordinate $u$ on $\mathbb{P}(F)$ is

$$
\operatorname{Res}_{\phi}\left(\vec{D}_{\infty}\right)_{H C}=\frac{2 \pi}{i} u \frac{\partial}{\partial u}\llcorner T
$$

where

$$
T=\int_{0}^{1} \phi\left(\frac{u^{*} D u}{|u|^{2}} ; \Lambda(x)\right) d x .
$$

Therefore, the residue form is independent of the choice of $\varepsilon$-circle bundle and hermitian metric on $\mathcal{R}$.

(2) The residue form $\operatorname{Res}_{\phi}\left(\vec{D}_{\infty}\right)$ is d-closed. 
Proof.

(1) Let $[t, u]$ be homogeneous local coordinates on $\mathbb{P}(E \oplus F)$. By (3.2),

$$
\left(T_{\infty, 0}\right)_{H C}=T+\frac{1}{2 \pi i} \frac{d t}{t}\left(\frac{2 \pi}{i} u \frac{\partial}{\partial u}\llcorner T) .\right.
$$

So,

$$
\begin{aligned}
\operatorname{Res}_{\phi}\left(\vec{D}_{\infty}\right)_{H C} & =\int_{\rho^{-1}}\left(T_{\infty, 0}\right)_{H C} \\
& =\left(\frac{1}{2 \pi i} \int_{|t|=\varepsilon} \frac{d t}{t}\right) \frac{2 \pi}{i} u \frac{\partial}{\partial u}\left\llcorner T=\frac{2 \pi}{i} u \frac{\partial}{\partial u}\llcorner T,\right.
\end{aligned}
$$

since $t$ can be regarded as a local fibre variable on the bundle $\pi: \mathcal{R} \rightarrow \mathbb{P}(F)$.

(2) It suffices to show that $\rho_{*}\left(d T_{\infty, 0}\right)=0$. Now $d T_{\infty, 0}=\phi\left(D_{F}\right)-\phi\left(\vec{D}_{0}\right)$ on $S_{\varepsilon}(\mathcal{R})$, since $S_{\varepsilon}(\mathcal{R}) \subset \operatorname{Hom}(E, F) \sim X$. By [HL, III.6.4], $\phi\left(\vec{D}_{0}\right)=\phi\left(\vec{\Omega}_{0}\right)$ where

$$
\vec{\Omega}_{0}=\left(1-\frac{u^{*} u}{|u|^{2}}\right)\left(\Omega_{F}-\frac{D u^{*} D u}{|u|^{2}}\right)+\frac{u^{*} \Omega_{E} u}{|u|^{2}} \quad \text { on } \operatorname{Hom}(E, F) .
$$

Furthermore, by [Z1, I.3.7],

$$
u \frac{\partial}{\partial u}\left\llcorner\phi ( \vec { D } _ { 0 } ) = 0 \quad \text { and } \quad u ^ { * } \frac { \partial } { \partial u ^ { * } } \left\llcorner\phi\left(\vec{D}_{0}\right)=0 .\right.\right.
$$

So, by Lemma 2.29 , the expressions for $\phi\left(D_{F}\right)$ and $\phi\left(\vec{D}_{0}\right)$ in homogeneous coordinates are of degree zero in the fibre differentials $d t, d \bar{t}$ of the bundle $p: \mathcal{R} \rightarrow \mathbb{P}(F)$. This implies that

$$
\rho_{*}\left(d T_{\infty, 0}\right)=\int_{\rho^{-1}} \phi\left(D_{F}\right)-\int_{\rho^{-1}} \phi\left(\vec{D}_{0}\right)=0 .
$$

Finally, we come to the main result of the paper.

Theorem 3.7. Let $\nu$ be an atomic section of $\mathbb{P}(E \oplus F) \rightarrow X$. Then the singular part of the Chern current $\phi\left(\left(\vec{D}_{\infty}\right)\right)$ is given by

$$
S=\nu^{*}\left(\operatorname{Res}_{\phi}\left(\vec{D}_{\infty}\right)\right) \operatorname{Div}_{\infty}(\nu) .
$$

Therefore,

$$
\phi\left(\left(\vec{D}_{\infty}\right)\right)=\phi\left(D_{F}\right)+\nu^{*}\left(\operatorname{Res}_{\phi}\left(\vec{D}_{\infty}\right)\right) \operatorname{Div}_{\infty}(\nu)
$$

and

$$
\begin{aligned}
d T_{\infty, 0}=\phi\left(D_{F}\right)+\nu^{*}\left(\operatorname{Res}_{\phi}\left(\vec{D}_{\infty}\right)\right) & \operatorname{Div}_{\infty}(\nu) \\
& -\phi\left(\vec{D}_{0}\right)-\operatorname{Res}_{\phi}\left(\vec{D}_{0}\right) \operatorname{Div}_{0}(\nu)
\end{aligned}
$$

on $X$. 
Note. The singular part of the Chern current at time infinity decomposes into the product of two terms - the pole divisor, which is independent of the invariant polynomial $\phi$, and the smooth form $\nu^{*}\left(\operatorname{Res}_{\phi}\left(\vec{D}_{\infty}\right)\right)$ on $X \sim Z$, which depends on both $\nu$ and $\phi$. This phenomenon should be compared and contrasted to the decomposition of the singular part of the Chern current at time zero, where the residue form $\operatorname{Res}_{\phi}\left(\vec{D}_{0}\right)$ on $X$ is $\nu$-independent.

\section{Corollary 3.11.}

$$
\operatorname{Res}_{c_{1}}\left(\vec{D}_{\infty}\right)=1
$$

so that

$$
c_{1}\left(\left(\vec{D}_{\infty}\right)\right)=c_{1}\left(D_{F}\right)+\operatorname{Div}_{\infty}(\nu)
$$

on $X$.

Remark 3.12. In the case $\operatorname{rank} F=1$, the Chern current formulae are particularly simple. Let $\phi=c_{1}^{m}$. Then,

$$
\begin{aligned}
d T_{\infty, 0}=c_{1}\left(D_{F}\right)^{m}-c_{1}\left(D_{E}\right)^{m} & \\
& +\left(\operatorname{Div}_{\infty}(\nu)-\operatorname{Div}_{0}(\nu)\right) \frac{c_{1}\left(D_{F}\right)^{m}-c_{1}\left(D_{E}\right)^{m}}{c_{1}\left(D_{F}\right)-c_{1}\left(D_{E}\right)}
\end{aligned}
$$

on $X$. The case $m=1$ gives a $C^{\infty}$-generalization of the classical Poincaré-Lelong formula,

$$
d T_{\infty, 0}=c_{1}\left(D_{F}\right)-c_{1}\left(D_{E}\right)+\operatorname{Div}_{\infty}(\nu)-\operatorname{Div}_{0}(\nu) .
$$

More generally, it was observed in [HL, II.9.7] that these formulae hold for sections $s$ of $F$, which can be expressed locally as $s=(a / b) f$, where $a, b: X \rightarrow \mathbb{C}$ are weakly atomic. Note that when $F \rightarrow X$ is a holomorphic line bundle over a Riemann surface, all such "meromorphic" sections $s$ of $F$ are holomorphic sections of $\mathbb{P}(\underline{\mathbb{C}} \oplus F)$ and vice-versa.

Proof of Theorem 3.7. We compute $S$ by calculating $d T_{\infty, 0}$ in each of the charts $\nu^{-1}\left(\mathcal{U}_{j}\right)$ By (3.2) and Lemma 3.6,

$$
T_{\infty, 0}=T+\frac{1}{2 \pi i} \frac{d t}{t} \nu^{*}\left(\operatorname{Res}_{\phi}\left(\vec{D}_{\infty}\right)\right) \quad \text { on } \nu^{-1}\left(\mathcal{U}_{j}\right)
$$

where $T$ and $\operatorname{Res}_{\phi}\left(\vec{D}_{\infty}\right)$ are smooth forms on $\nu^{-1}\left(\mathcal{U}_{j}\right)$. So,

$$
d T_{\infty, 0}=d T+\nu^{*}\left(\operatorname{Res}_{\phi}\left(\vec{D}_{\infty}\right)\right) \operatorname{Div}_{\infty}(\nu) \quad \text { on } \nu^{-1}\left(\mathcal{U}_{j}\right),
$$

since $d \operatorname{Res}_{\phi}\left(\vec{D}_{\infty}\right)=0$. On the other hand, we know that

$$
d T_{\infty, 0}=\phi\left(D_{F}\right)+S-\phi\left(\vec{D}_{0}\right)
$$


where $\phi\left(\vec{D}_{0}\right)$ and $\phi\left(D_{F}\right)$ are smooth forms on $\nu^{-1}\left(\mathcal{U}_{j}\right)$. So,

$$
S-\nu^{*}\left(\operatorname{Res}_{\phi}\left(\vec{D}_{\infty}\right)\right) \operatorname{Div}_{\infty}(\nu)=\phi\left(\vec{D}_{0}\right)-\phi\left(D_{F}\right)+d T,
$$

and since the L.H.S. is zero on the dense subset $\nu^{-1}\left(\mathcal{U}_{j}\right) \sim P$ and the R.H.S. is smooth on $\nu^{-1}\left(\mathcal{U}_{j}\right)$, we are forced to conclude that both sides of the equation are zero on $\nu^{-1}\left(\mathcal{U}_{j}\right)$. This proves that $S=\nu^{*}\left(\operatorname{Res}_{\phi}\left(\vec{D}_{\infty}\right)\right) \operatorname{Div}_{\infty}(\nu)$ on $\nu^{-1}\left(\mathcal{U}_{j}\right)$ and hence on all of $X$.

Remark 3.15. The pullback case. Analogous results hold in the pullback case, with $\phi=c^{-1}$. In particular,

$$
\left(T_{r, s}\right)_{H C}=T_{r, s}-\frac{d t}{t}\left(u \frac{\partial}{\partial u}\left\llcorner T_{r, s}\right)\right.
$$

where

$$
\begin{aligned}
T_{r, s}:=\frac{i}{2 \pi} \frac{D u u^{*}}{|u|^{2}} \frac{\left(c\left(D_{E}\right)+\chi_{r}\left(c\left(\overleftarrow{D}_{0}\right)-c\left(D_{E}\right)\right)\right)^{-1}}{c\left(\overleftarrow{D}_{0}\right)-c\left(D_{E}\right)} \\
-\frac{\left(c\left(D_{E}\right)+\chi_{s}\left(c\left(\overleftarrow{D}_{0}\right)-c\left(D_{E}\right)\right)\right)^{-1}}{c\left(\overleftarrow{D}_{0}\right)-c\left(D_{E}\right)}
\end{aligned}
$$

and $c\left(\overleftarrow{D}_{0}\right)=c\left(\overleftarrow{\Omega}_{0}\right)$ where

$$
\overleftarrow{\Omega}_{0}=\frac{u \Omega_{F} u^{*}}{|u|^{2}}-\frac{D u\left(1-\frac{u^{*} u}{|u|^{2}}\right) D u^{*}}{|u|^{2}}
$$

The transgression current $T_{\infty, 0}=\underset{\substack{\lim \rightarrow \rightarrow 0 \\ r \rightarrow \infty}}{ } T_{r, s}$ converges in $L_{\text {loc }}^{1}(X)$ and equals

$$
\left(T_{\infty, 0}\right)_{H C}=T-\frac{d t}{t}\left(u \frac{\partial}{\partial u}\llcorner T)\right.
$$

where

$$
T=-\frac{i}{2 \pi} \frac{D u u^{*}}{|u|^{2}} \frac{c\left(D_{E}\right)^{-1}-c\left(\overleftarrow{D}_{0}\right)^{-1}}{c\left(D_{E}\right)-c\left(\overleftarrow{D}_{0}\right)}
$$

The Chern current at time infinity exists and is given by

$$
c^{-1}\left(\left(\overleftarrow{D}_{\infty}\right)\right)=c\left(D_{E}\right)^{-1}+c\left(D_{E}\right)^{-1} \nu^{*}\left(c\left(D_{L}\right)^{-1}\right) \operatorname{Div}_{\infty}(\nu)
$$


where $L$ is the tautological line bundle on $\mathbb{P}(F)$ with connection $D_{L}$ induced from that on $F$. Furthermore,

$$
\begin{aligned}
d T_{\infty, 0}=c\left(D_{E}\right)^{-1}+c\left(D_{E}\right)^{-1} \nu^{*}\left(c\left(D_{L}\right)^{-1}\right) \operatorname{Div}_{\infty}(\nu) \\
-c^{-1}\left(\overleftarrow{D}_{0}\right)-c\left(D_{E}\right)^{-1} c\left(D_{F}\right)^{-1} \operatorname{Div}_{0}(\nu)
\end{aligned}
$$

Note that,

$$
\operatorname{Res}_{c^{-1}}\left(\overleftarrow{D}_{\infty}\right)=c\left(D_{E}\right)^{-1} c\left(D_{L}\right)^{-1}
$$

and

$$
\operatorname{Res}_{c^{-1}}\left(\overleftarrow{D}_{0}\right)=c\left(D_{E}\right)^{-1} c\left(D_{F}\right)^{-1}
$$

on $X$.

Also note that in the case $\operatorname{rank} F=1$, equation (3.16) reduces to the negative of equation (3.13).

Note. These results are proved just as in the pushforward case. To prove (3.17) note that $\left(U_{0}, \overleftarrow{D}_{0}\right)=\left(L, D_{L}\right)$ on the projective space $\mathbb{P}(F)$ at infinity

4. Residues at infinity. In this section we aim to compute the residue form at infinity, $\operatorname{Res}_{\phi}\left(\vec{D}_{\infty}\right)$, on the projective bundle $\mathbb{P}(F)$ at infinity in $\mathbb{P}(E \oplus F)$. Except for the following fact, our results are entirely analogous to those of Harvey and Lawson [HL, III.7]. They were able to prove that the residue form $\operatorname{Res}_{\phi}\left(\vec{D}_{0}\right)$ on $X$ is a polynomial $\psi\left(c_{1}\left(D_{E}\right), c_{1}\left(D_{F}\right), \ldots, c_{n}\left(D_{F}\right)\right)$ in the Chern forms of the bundles $\left(E, D_{E}\right)$ and $\left(F, D_{F}\right)$. In contrast to this, the residue form at infinity on $\mathbb{P}(F)$ is given by

$$
\operatorname{Res}_{\phi}\left(\vec{D}_{\infty}\right)=\psi\left(c_{1}\left(D_{L}\right), c_{1}\left(D_{E}\right), c_{1}\left(D_{F}\right), \ldots, c_{n}\left(D_{F}\right)\right)+d S_{\phi},
$$

where $L \rightarrow \mathbb{P}(F)$ is the tautological line bundle and $\psi$ is a polynomial in the Chern forms of the bundles $\left(E, D_{E}\right),\left(F, D_{F}\right)$ and $\left(L, D_{L}\right)$. The important point here is that the differential form $S_{\phi}$ is not closed in general. Therefore, we often prefer to compute the residue class, $\left[\operatorname{Res}_{\phi}\left(\vec{D}_{\infty}\right)\right]$, rather than the residue form itself.

The residue class is computable in the sense that, for any invariant polynomial $\phi$, there is an explicit formula for the polynomial $\psi$ for which

$$
\left[\operatorname{Res}_{\phi}\left(\vec{D}_{\infty}\right)\right]=\psi\left(c_{1}(L), c_{1}(E), c_{1}(F), \ldots, c_{n}(F)\right) .
$$

Here $c_{j}(V)$ denotes the $j^{\text {th }}$ Chern class of the bundle $V$. Actually, for any given invariant polynomial $\phi$, it is also possible to explicitly calculate the form $S_{\phi}$ in a local homogeneous coordinate system on $\mathbb{P}(F)$. In particular we will prove that $S_{c_{1}^{m}}$ and $S_{c_{2}}$ are both zero. However, this behaviour is far from typical as is suggested by the example of $S_{c_{3}}$, which is not even a closed form on $\mathbb{P}(F)$. The following analogue of [HL, III.2.24] provides an alternate definition of the residue form at infinity. 
Theorem 4.1. Let $\pi: \mathbb{P}(E \oplus F) \sim X \rightarrow \mathbb{P}(F)$ be defined by (1.7). Then, for any invariant polynomial $\phi$,

$$
\operatorname{Res}_{\phi}\left(\vec{D}_{\infty}\right)=\pi_{*}\left(\phi\left(D_{Q}\right)\right)=\int_{\pi^{-1}} \phi\left(D_{Q}\right) .
$$

Proposition 4.2. Consider the bundle map $\eta: \mathbb{P}(F) \rightarrow X$. Then

$$
\operatorname{Res}_{\phi}\left(\vec{D}_{0}\right)=\int_{\eta-1} \operatorname{Res}_{\phi}\left(\vec{D}_{\infty}\right)
$$

Proof. Recall [HL, III.7.6] that the residue form on $X$ is given by the fibre integral

$$
\begin{aligned}
\operatorname{Res}_{\phi}\left(\vec{D}_{0}\right) & =\int_{\mathbb{P}(E \oplus F)_{x}} \phi\left(D_{Q}\right)=\int_{\mathcal{R}_{x}} \phi\left(D_{Q}\right)=\int_{\mathbb{P}(F)_{x}} \int_{\pi^{-1}} \phi\left(D_{Q}\right) \\
& =\int_{\mathbb{P}(F)_{x}} \operatorname{Res}_{\phi}\left(\vec{D}_{\infty}\right)=\int_{\eta^{-1}} \operatorname{Res}_{\phi}\left(\vec{D}_{\infty}\right) .
\end{aligned}
$$

The next result will be used to calculate the residue class at infinity. It is proved by combining the analogue of Theorem 4.1 in the pullback case with equation (3.17).

Proposition 4.3. Let $\pi: \mathbb{P}(E \oplus F) \sim X \rightarrow \mathbb{P}(F)$ be as above. Then

$$
\pi_{*}\left(c\left(D_{U}\right)^{-1}\right)=\int_{\pi^{-1}} c\left(D_{U}\right)^{-1}=c\left(D_{E}\right)^{-1} c\left(D_{L}\right)^{-1} .
$$

In particular,

$$
\pi_{*}\left(c_{1}^{k}\left(D_{U}\right)\right)=-\frac{c_{1}^{k}\left(D_{L}\right)-c_{1}^{k}\left(D_{E}\right)}{c_{1}\left(D_{L}\right)-c_{1}\left(D_{E}\right)}=-\sum_{i+j=k-1} c_{1}^{i}\left(D_{E}\right) c_{1}^{j}\left(D_{L}\right) .
$$

We are now in a position to calculate the residue class $\left[\operatorname{Res}_{\phi}\left(\vec{D}_{\infty}\right)\right]$ for an arbitrary invariant polynomial $\phi$. Recall that any $\mathfrak{g l}(n, \mathbb{C})$ invariant polynomial can be expressed as a polynomial in $c_{1}, \ldots, c_{n}$ and that the residue of a sum of polynomials is the sum of the residues. Consequently, to compute the residue for a general $\phi$ it suffices to compute it for the polynomials,

$$
\phi=\prod_{k=1}^{n} c_{k}^{j_{k}}
$$


Since

$$
0 \rightarrow U \rightarrow \mathbf{E} \oplus \mathbf{F} \rightarrow Q \rightarrow 0
$$

is exact over $\mathbb{P}(E \oplus F)$, we have the sequence of formulae

$$
\begin{gathered}
c(E \oplus F)=c(U \oplus Q), \\
c(E) c(F)=c(U) c(Q), \\
c(Q)=c(U)^{-1} c(E) c(F), \quad \text { and } \\
c_{k}(Q)=\left\{c(U)^{-1} c(E) c(F)\right\}_{k},
\end{gathered}
$$

where $\{T\}_{k}$ denotes the degree $2 k$ part of $T$. Therefore, for any $\phi$ given by (4.4), we have

$$
\begin{aligned}
\phi(Q) & =\prod_{k=1}^{n}\left(\left\{c(U)^{-1} c(E) c(F)\right\}_{k}\right)^{j_{k}} \\
& =\sum_{\ell=0}^{\infty} c_{1}^{\ell}(U) \psi_{\ell}\left(c_{1}(E), c_{1}(F), \ldots, c_{n}(F)\right),
\end{aligned}
$$

where the polynomials $\psi_{\ell}$ are defined by this last equation. Applying Theorem 4.1 and Proposition 4.3, we have the following result.

Theorem 4.11. Let $\phi=\prod_{k=1}^{n} c_{k}^{j_{k}}$. Then

$$
\left[\operatorname{Res}_{\phi}\left(\vec{D}_{\infty}\right)\right]=-\sum_{\ell=0}^{\infty} \frac{c_{1}^{\ell}(L)-c_{1}^{\ell}(E)}{c_{1}(L)-c_{1}(E)} \psi_{\ell}\left(c_{1}(E), c_{1}(F), \ldots, c_{n}(F)\right)
$$

in $H^{*}(\mathbb{P}(F) ; \mathbb{R})$, where $\psi_{\ell}$ is the polynomial defined by equation (4.10). In particular,

(1) $\left[\operatorname{Res}_{c}\left(\vec{D}_{\infty}\right)\right]=c(L)^{-1} c(F)$.

(2) $\operatorname{Res}_{c_{1}}\left(\vec{D}_{\infty}\right)=1$.

(3) Let $T=-c_{1}(L)$. Then, for $k \in\{2, \ldots, n\}$,

$$
\left[\operatorname{Res}_{c_{k}}\left(\vec{D}_{\infty}\right)\right]=T^{k-1}+T^{k-2} c_{1}(F)+\cdots+c_{k-1}(F) .
$$




$$
\begin{gathered}
{\left[\operatorname{Res}_{\mathrm{ch}}\left(\vec{D}_{\infty}\right)\right]=\sum_{k=1}^{\infty} \frac{1}{k !} \frac{c_{1}^{k}(L)-c_{1}^{k}(E)}{c_{1}(L)-c_{1}(E)}} \\
{\left[\operatorname{Res}_{b_{k}}\left(\vec{D}_{\infty}\right)\right]=\frac{c_{1}^{k}(L)-c_{1}^{k}(E)}{c_{1}(L)-c_{1}(E)}}
\end{gathered}
$$

where the $k^{\text {th }}$ trace power $b_{k}$ is defined by $b_{k}(\Omega):=\operatorname{tr}\left((i \Omega / 2 \pi)^{k}\right)$.

(6) $\left[\operatorname{Res}_{c^{-1}}\left(\vec{D}_{\infty}\right)\right]=-c(E)^{-1} c(F)^{-1}$.

(7) Let $\mathbb{H}(c)$ be a multiplicative series of Chern classes associated to the formal power series $h(x)=1+a_{1} x+a_{2} x^{2}+\cdots \in \mathbb{R}[x]$. Then,

$$
\left[\operatorname{Res}_{\mathbb{H}}\left(\vec{D}_{\infty}\right)\right]=\pi_{!}\left(h^{-1}\left(c_{1}(U)\right)\right) \mathbb{H}(E) \mathbb{H}(F)
$$

In particular, if $h^{-1}(x)=1+b_{1} x+b_{2} x^{2}+\cdots$ is the formal power series for the reciprical of $h$, then

$$
\left[\operatorname{Res}_{\mathbb{H}}\left(\vec{D}_{\infty}\right)\right]=-\sum_{k=1}^{\infty} b_{k} \frac{c_{1}^{k}(L)-c_{1}^{k}(E)}{c_{1}(L)-c_{1}(E)} \mathbb{H}(E) \mathbb{H}(F)
$$

Remark 4.12. See $[\mathrm{LM}]$ for the definition of a multiplicative series of Chern classes. The main examples are given by the Todd series $\mathbb{H}=\mathrm{Td}$ in which case $h(x)=x /\left(1-e^{-x}\right)$ and the series $\mathbb{H}=e^{c_{1}}$ generated by $h(x)=e^{x}$.

Note. The seven formulae above can be proved directly using the exact sequence (4.5), Proposition 4.3 and the properties of the particular invariant polynomials under consideration.

Remark 4.13. Flat case. Let $T=-c_{1}(L)$. Then, in the flat case, we have

$$
\begin{aligned}
& {\left[\operatorname{Res}_{c}\left(\vec{D}_{\infty}\right)\right]=c(L)^{-1},} \\
& {\left[\operatorname{Res}_{\mathrm{ch}}\left(\vec{D}_{\infty}\right)\right]=\operatorname{Td}^{-1}(T),} \\
& {\left[\operatorname{Res}_{b_{k}}\left(\vec{D}_{\infty}\right)\right]=b_{k-1}(L),} \\
& {\left[\operatorname{Res}_{c^{-1}}\left(\vec{D}_{\infty}\right)\right]=0,} \\
& {\left[\operatorname{Res}_{\mathbb{H}}\left(\vec{D}_{\infty}\right)\right]=-\sum_{k=1}^{n} b_{k} c_{1}^{k-1}(L),}
\end{aligned}
$$


and for any degree $m$ invariant polynomial $\phi$ which is a simple product of the Chern polynomials $c_{1}, \ldots, c_{n}$,

$$
\left[\operatorname{Res}_{\phi}\left(\vec{D}_{\infty}\right)\right]=T^{m-1} .
$$

In particular,

$$
\left[\operatorname{Res}_{\phi}\left(\vec{D}_{\infty}\right)\right]=0 \quad \text { for degree } \phi>n .
$$

Actually, the next proposition shows that these formulae hold on the form level.

Proposition 4.14. Let $\phi=\prod_{k=1}^{n} c_{k}^{j_{k}}$ be an invariant polynomial of degree $m$. Then, in the flat case,

$$
\operatorname{Res}_{\phi}\left(\vec{D}_{\infty}\right)=\left(-c_{1}\left(D_{L}\right)\right)^{m-1} .
$$

Proof. Let $u$ be a homogeneous coordinate on $\mathbb{P}(F)$. First note that

$$
b_{k}\left(d u^{*} d u\right)=\operatorname{tr}\left(\left(\frac{i}{2 \pi} d u^{*} d u\right)^{k}\right)=-\left(\frac{i}{2 \pi} d u d u^{*}\right)^{k} .
$$

Then, by the Newton identities,

$$
c_{k}\left(-d u^{*} d u\right)=\left(\frac{i}{2 \pi} d u d u^{*}\right)^{k}
$$

and so

$$
\phi\left(-d u^{*} d u\right)=\left(\frac{i}{2 \pi} d u d u^{*}\right)^{m} .
$$

So, by Lemma 3.6,

$$
\begin{aligned}
\operatorname{Res}_{\phi}\left(\vec{D}_{\infty}\right) & =-\frac{1}{m}\left(\frac{i}{2 \pi}\right)^{m-1} u \frac{\partial}{\partial u}\left\llcorneru ^ { * } \frac { \partial } { \partial u ^ { * } } \left\llcorner\left(\frac{d u d u^{*}}{|u|^{2}}\right)^{m}\right.\right. \\
& =\left(\frac{i}{2 \pi}\right)^{m-1}\left(\frac{d u d u^{*}}{|u|^{2}}-\frac{d u u^{*} u d u^{*}}{|u|^{4}}\right)^{m-1} \\
& =\left(-c_{1}\left(D_{L}\right)\right)^{m-1} .
\end{aligned}
$$

Next we state a formula which relates $\left[\operatorname{Res}_{c_{1} \psi}\left(\vec{D}_{\infty}\right)\right]$ to $\left[\operatorname{Res}_{\psi}\left(\vec{D}_{\infty}\right)\right]$. Its proof is analogous to [HL, III.4.16] which states that $\operatorname{Res}_{c_{n} \psi}\left(\vec{D}_{0}\right)=\psi\left(D_{F}\right)$ on $X$. 
Proposition 4.15. Let $\psi$ be any invariant polynomial. Then,

$$
\left[\operatorname{Res}_{c_{1} \psi}\left(\vec{D}_{\infty}\right)\right]=c_{1}(F)\left[\operatorname{Res}_{\psi}\left(\vec{D}_{\infty}\right)\right]+\psi\left(E \oplus L^{\perp}\right)
$$

Finally we state some results concerning residue forms (rather than residue classes) on $\mathbb{P}(F)$.

Theorem 4.16.

$$
\operatorname{Res}_{c_{1}^{m}}\left(\vec{D}_{\infty}\right)=\frac{\left(c_{1}\left(D_{F}\right)+c_{1}\left(D_{E}\right)-c_{1}\left(D_{L}\right)\right)^{m}-c_{1}\left(D_{F}\right)^{m}}{c_{1}\left(D_{E}\right)-c_{1}\left(D_{L}\right)}
$$

as differential forms on $\mathbb{P}(F)$.

Proof. Let $u$ be a homogeneous coordinate on $\mathbb{P}(F)$. Either by direct calculation or using [HL, III.7.33] we have

$$
c_{1}\left(D_{L}\right)=\frac{i}{2 \pi}\left(\frac{u \Omega_{F} u^{*}}{|u|^{2}}-\frac{D u\left(1-\frac{u^{*} u}{|u|^{2}}\right) D u^{*}}{|u|^{2}}\right) .
$$

Then, by Lemma 3.6,

$$
\begin{aligned}
\operatorname{Res}_{c_{1}^{m}}\left(\vec{D}_{\infty}\right) & =-\left(\frac{i}{2 \pi}\right)^{m-1} u \frac{\partial}{\partial u}\left\llcorner u^{*} \frac{\partial}{\partial u^{*}}\right. \\
& \left\llcorner\int_{0}^{1} \operatorname{tr}^{m}\left(\Omega_{F}-x\left(\frac{u^{*} u}{|u|^{2}}\left(\Omega_{F}-\Omega_{E}\right)+\frac{D u^{*} D u}{|u|^{2}}\right)\right) \frac{d x}{x}\right. \\
= & -\left(\frac{i}{2 \pi}\right)^{m-1} u \frac{\partial}{\partial u}\left\llcorner u^{*} \frac{\partial}{\partial u^{*}}\right. \\
= & \int_{0}^{1} m\left(c_{1}^{1}\left(D_{F}\right)+x\left(c_{1}\left(\Omega_{F}\right)+x \Omega_{E}-x \frac{u \Omega_{F} u^{*}}{|u|^{2}}+x \frac{D u D u^{*}}{|u|^{2}}\right)^{m} \frac{d x}{x}\right. \\
= & \frac{\left.\left(c_{1}\left(D_{F}\right)\right)^{m-1}\right) d x}{c_{1}\left(D_{E}\right)-c_{1}\left(D_{L}\right)}
\end{aligned}
$$




\section{Theorem 4.17.}

$$
\begin{aligned}
& \operatorname{Res}_{c_{2}}\left(\vec{D}_{\infty}\right)=c_{1}\left(D_{F}\right)-c_{1}\left(D_{L}\right), \\
& \operatorname{Res}_{b_{2}}\left(\vec{D}_{\infty}\right)=c_{1}\left(D_{E}\right)+c_{1}\left(D_{L}\right), \\
& \operatorname{Res}_{b_{3}}\left(\vec{D}_{\infty}\right)=c_{1}^{2}\left(D_{E}\right)+c_{1}\left(D_{E}\right) c_{1}\left(D_{L}\right)+c_{1}^{2}\left(D_{L}\right)+d S, \\
& \operatorname{Res}_{c_{3}}\left(\vec{D}_{\infty}\right)=c_{2}\left(D_{F}\right)-c_{1}\left(D_{L}\right) c_{1}\left(D_{F}\right)+c_{1}^{2}\left(D_{L}\right)+\frac{1}{3} d S,
\end{aligned}
$$

where

$$
S=-\frac{3}{8 \pi} \frac{i}{2 \pi} \frac{D u\left(1-\frac{u^{*} u}{|u|^{2}}\right) \Omega_{F} u^{*}}{|u|^{2}}
$$

in a homogeneous coordinate $u$ on $\mathbb{P}(F)$, and

$$
d S \not \equiv 0 .
$$

Proof. For any $\mathfrak{g l}(n+1, \mathbb{C})$ invariant polynomial $\phi$, there is a smooth transgression form $S_{\phi}$ so that $\phi\left(D_{E} \oplus D_{F}\right)-\phi\left(D_{U} \oplus D_{Q}\right)=d S_{\phi}$ on $\mathbb{P}(E \oplus F)$. In particular,

$$
b_{k}\left(D_{Q}\right)=b_{k}\left(D_{E}\right)+b_{k}\left(D_{F}\right)-b_{k}\left(D_{U}\right)-d S_{b_{k}}
$$

and so,

$$
\begin{aligned}
\operatorname{Res}_{b_{k}}\left(D_{Q}\right) & =-\pi_{*}\left(b_{k}\left(D_{U}\right)\right)-d \pi_{*}\left(S_{b_{k}}\right) \\
& =-\pi_{*}\left(c_{1}^{k}\left(D_{U}\right)\right)-d \pi_{*}\left(S_{b_{k}}\right) \\
& =\frac{c_{1}^{k}\left(D_{L}\right)-c_{1}^{k}\left(D_{E}\right)}{c_{1}\left(D_{L}\right)-c_{1}\left(D_{E}\right)}-d \pi_{*}\left(S_{b_{k}}\right) .
\end{aligned}
$$

So to prove (4.19) and (4.20), it suffices to show that

$$
\pi_{*}\left(S_{b_{2}}\right)=0 \quad \text { and } \quad \pi_{*}\left(S_{b_{3}}\right)=S .
$$

Note that (4.18) follows from (4.19) and Proposition 4.16 using the Newton identity,

$$
c_{2}=\frac{1}{2}\left(c_{1}^{2}-b_{2}\right)
$$

We will discuss the proof of (4.21) at the end.

To prove that $\pi_{*}\left(S_{b_{2}}\right)=0$, we use [HL, III.7.35] to derive the formula

$$
S_{b_{2}}=-\frac{1}{2 \pi^{2}}\left(\frac{D u \Omega_{F} u^{*}}{|u|^{2}+|t|^{2}}-\frac{D u u^{*}}{|u|^{2}+|t|^{2}} \frac{|t|^{2} \Omega_{E}+u \Omega_{F} u^{*}}{|u|^{2}+|t|^{2}}+\frac{\bar{t} d t\left(|u|^{2} \Omega_{E}-u \Omega_{F} u^{*}\right)}{\left(|u|^{2}+|t|^{2}\right)^{2}}\right)
$$


in homogeneous coordinates $[t, u]$ on $\mathbb{P}(E \oplus F)$, and note that since $S_{b_{2}}$ has no terms of degree 2 in $d t, d \bar{t}$,

$$
\pi_{*}\left(S_{b_{2}}\right)=\int_{\pi^{-1}} S_{b_{2}}=0
$$

Similarly the part of $S_{b_{3}}$ which is of degree 2 in $d t, d \bar{t}$ is given by

$$
-3\left(\frac{i}{2 \pi}\right)^{3} \frac{d t d \bar{t}}{|t|^{2}} u \frac{\partial}{\partial u}\left\llcorner u^{*} \frac{\partial}{\partial u^{*}}\llcorner B\right.
$$

where

$$
\begin{gathered}
B=\chi \frac{D u \Omega_{F} u^{*}}{|u|^{2}}-\chi^{2} \frac{D u u^{*}\left(\Omega_{E}^{2}+u \Omega_{F} u^{*}\right)}{|u|^{4}} \\
-\chi^{2} \frac{D u \Omega_{F} u^{*}}{|u|^{2}}\left(\frac{D u D u^{*}}{|u|^{2}}-\chi \frac{D u u^{*} u D u^{*}}{|u|^{4}}\right) \\
+\chi^{3} \frac{D u u^{*}\left(\Omega_{E}+u \Omega_{F} u^{*}\right) D u D u^{*}}{|u|^{6}}
\end{gathered}
$$

and $\chi:=|u|^{2} /\left(|u|^{2}+|t|^{2}\right)$. Consequently,

$$
\begin{aligned}
\pi_{*}\left(S_{b_{3}}\right) & =3\left(\frac{i}{2 \pi}\right)^{3} \int_{\pi^{-1}} \chi^{2}(1-\chi)\left(\frac{u \Omega_{F} u^{*} D u u^{*}}{|u|^{4}}-\frac{D u \Omega_{F} u^{*}}{|u|^{2}}\right) \frac{d t d \bar{t}}{|t|^{2}} \\
& =3\left(\frac{i}{2 \pi}\right)^{3}\left(\frac{D u \Omega_{F} u^{*}}{|u|^{2}}-\frac{D u u^{*} u \Omega_{F} u^{*}}{|u|^{4}}\right) \int_{\pi^{-1}} \frac{|u|^{4} d t d \bar{t}}{\left(|t|^{2}+|u|^{2}\right)^{3}} \\
& =S .
\end{aligned}
$$

Finally, to prove (4.21) we use the Newton identity,

$$
6 c_{3}=2 b_{3}-3 b_{1} b_{2}+b_{1}^{3},
$$

and check that

$$
\pi_{*}\left(S_{b_{1} b_{2}}\right)=b_{1}\left(\Omega_{E \oplus F}\right) \pi_{*}\left(S_{b_{2}}\right)=0 .
$$

5. The relationship to Levine's construction. Levine $[\mathrm{L}]$ studied the Chern current $c_{1}^{m}\left(\left(\overleftarrow{D}_{\pi, 0}\right)\right)$ at time zero of the natural projection $\pi: U \rightarrow E$ over $\mathbb{P}(E \oplus F)$. This construction was subsequently examined by Gillet and Soulé [GS] and by Harvey and Lawson [HL, II.10]. In this section we examine the relationship between the families of connections $D_{U, r}$ and $\overleftarrow{D}_{\pi, s}$ and also between $D_{Q, r}$ and $\vec{D}_{\pi, s}$. Our main result is that in the pullback case the Chern current 
$c_{1}^{m}\left(\left(\overleftarrow{D}_{\alpha, \infty}\right)\right)$ at time infinity of the tautological homomorphism $\alpha$ is equal to the Chern current $c_{1}^{m}\left(\left(\overleftarrow{D}_{\pi, 0}\right)\right)$. Note that the zero set of the map $\pi$ is the pole set of $\alpha$. We also investigate the extent to which the pushforward analogue of this result holds. The discussion splits into four cases corresponding to the families of connections $D_{U, r}$ and $D_{Q, r}$ at times $r=0$ and $r=\infty$. We assume that rank $E=1$ unless otherwise stated.

Pullback at zero. Let $\pi: E \oplus F \rightarrow F$ and consider the blow up

$$
\widehat{\mathbb{P}}(E \oplus F)=\left\{(A, B) \in \mathbb{P}(E \oplus F) \times_{X} \mathbb{P}(F) / \pi(A) \subset B\right\} .
$$

Recall that the projection $p: \widehat{\mathbb{P}}(E \oplus F) \rightarrow \mathbb{P}(E \oplus F)$ onto the first factor is a diffeomorphism over $\mathbb{P}(E \oplus F) \sim X$ and that $p^{-1}(X)=\mathbb{P}(F)_{0}$ is a copy of $\mathbb{P}(F)$ over spatial zero. Let $\mathbf{U}$ and $\mathbf{L}$ be the pullbacks of the tautological line bundles $U \rightarrow \mathbb{P}(E \oplus F)$ and $L \rightarrow \mathbb{P}(F)$. The blow up $\widehat{\mathbb{P}}(E \oplus F)$ has been chosen so that the induced map $\pi: U \rightarrow L$ over $\mathbb{P}(E \oplus F) \rightarrow X$ desingularises to define an atomic bundle map $\pi: \mathbf{U} \rightarrow \mathbf{L}$ over $\widehat{\mathbb{P}}(E \oplus F)$, with $\operatorname{Div}(\pi)=\left[\mathbb{P}(F)_{0}\right]$. Let $\overleftarrow{D}_{\pi, s}$ be the pullback family of connections on $\mathbf{U}$ induced by $\pi$, using the algebraic approximation mode, and let $D_{\mathbf{U}, r}$ be the pullback to $\mathbf{U}$ of $D_{U, r}$ on $U$.

Theorem 5.1. Let $0<r<1$ and $0<s<\infty$ be related by $r=s /(1+s)$. Then

$$
D_{\mathbf{U}, r}=\overleftarrow{D}_{\pi, s} \quad \text { on } \mathbf{U} \text { over } \widehat{\mathbb{P}}(E \oplus F)
$$

Remark 5.2. Let $1 \leq p=\operatorname{rank} E \leq \operatorname{rank} F$ and consider the blow up $\widehat{G}_{p}(E \oplus F)$ defined as above. Let $U \rightarrow G_{p}(E \oplus F)$ and $L \rightarrow G_{p}(F)$ be the tautological rank $p$ bundles and define $\pi: \mathbf{U} \rightarrow \mathbf{L}$ as before. Then $D_{\mathbf{U}, r}=\overleftarrow{D}_{\pi, s}$ on $\mathbf{U}$.

Corollary 5.3. The current equation

$$
c_{1}^{m}\left(D_{\mathbf{U}}\right)-c_{1}^{m}\left(D_{\mathbf{L}}\right)-\operatorname{Res}_{m}\left(\overleftarrow{D}_{\pi, 0}\right)\left[\mathbb{P}(F)_{0}\right]=d T_{\infty, 0} \quad \text { on } \widehat{\mathbb{P}}(E \oplus F)
$$

pushes down via $p$ to the current equation

$$
c_{1}^{m}\left(\overleftarrow{D}_{\alpha, 1}\right)-c_{1}^{m}\left(\overleftarrow{D}_{\alpha, 0}\right)-\operatorname{Res}_{m}\left(\overleftarrow{D}_{\alpha, 0}\right)[X]=d T_{1,0} \quad \text { on } \mathbb{P}(E \oplus F)
$$

In particular, the residue $\operatorname{Res}_{m}\left(\overleftarrow{D}_{\alpha, 0}\right)$ of a map into a bundle of arbitrary rank is the fibre integral,

$$
\operatorname{Res}_{m}\left(\overleftarrow{D}_{\alpha, 0}\right)=\int_{\mathbb{P}(F)_{0}} \operatorname{Res}_{m}\left(\overleftarrow{D}_{\pi, 0}\right)
$$

of the residue $\operatorname{Res}_{m}\left(\overleftarrow{D}_{\pi, 0}\right)$ of a map between line bundles. 
Note. The fibre integral (5.4) is computed using Bott's Theorem (see [B] or [HL, III.7.28]).

Proof of Theorem 5.1. Let $\gamma_{s}: E \rightarrow U_{s}$ be the graphing map defined over $\operatorname{Hom}(E, F)$ by $\gamma_{s}(e)=(s e, \alpha(e))$ and let $\gamma:=\gamma_{1}$. It suffices to prove that $\gamma^{-1} \circ$ $\overleftarrow{D}_{\pi, s} \circ \gamma=\overleftarrow{D}_{\alpha, r}$ on $E$ over $\operatorname{Hom}(E, F)$. Since $\gamma^{-1} \pi^{*} \pi \gamma=\left(1+\alpha^{*} \alpha\right)^{-1} \alpha^{*} \alpha$ $\pi \gamma=\gamma_{0}$ and $\gamma_{s}^{-1} \circ D_{U_{s}} \circ \gamma_{s}=\overleftarrow{D}_{\alpha, s}$, we have that

$$
\begin{aligned}
\gamma^{-1} \circ \overleftarrow{D}_{\pi, s} \circ \gamma & =\gamma^{-1}\left(\pi^{*} \pi+s\right)^{-1}\left(s D_{U}+\pi^{*} D_{U_{0}} \pi\right) \gamma \\
& =\left(\left(1+\alpha^{*} \alpha\right)^{-1} \alpha^{*} \alpha+s\right)^{-1}\left(1+\alpha^{*} \alpha\right)^{-1}\left(s\left(D_{E}+\alpha^{*} D_{F} \alpha\right)+\alpha^{*} D_{F} \alpha\right) \\
& =\left(\frac{s}{1+s}+\alpha^{*} \alpha\right)^{-1}\left(\frac{s}{1+s} D_{E}+\alpha^{*} D_{F} \alpha\right)=\overleftarrow{D}_{\alpha, r}
\end{aligned}
$$

Pullback at infinity. Swapping the roles of $E$ and $F$ in the previous case, let $\pi: E \oplus F \rightarrow E$ and consider the induced map $\pi: U \rightarrow E$ over $\mathbb{P}(E \oplus F)$. Note that the mapping $\pi$ is atomic with $\operatorname{Div}(\pi)=[\mathbb{P}(F)]$ since $U \subset F$ on $\mathbb{P}(F)$.

Theorem 5.5. Let $0<s<\infty$ and $\infty>r>1$ be related by $r=(1+s) / s$. Then

$$
D_{U, r}=\overleftarrow{D}_{\pi, s} \quad \text { on } U \text { over } \mathbb{P}(E \oplus F)
$$

Remark 5.6. If $1 \leq p=\operatorname{rank} E \leq \operatorname{rank} F$, then $\overleftarrow{D}_{\pi, s}=D_{U, r}$ on $U$ over $G_{p}(E \oplus F)$.

Corollary 5.7. The Chern current $c_{1}^{m}\left(\left(\overleftarrow{D}_{\alpha, \infty}\right)\right)$ at time infinity of the tautological homomorphism $\alpha$ exists and equals the Chern current $c_{1}^{m}\left(\left(\overleftarrow{D}_{\pi, 0}\right)\right)$ at time zero of the map $\pi: U \rightarrow E$ of line bundles.

Proof of Theorem 5.5. Observe that $\gamma^{-1} \pi^{*} \pi \gamma=\left(1+\alpha^{*} \alpha\right)^{-1}$ and that $\pi \gamma=1$. The proof now follows along the same lines as that of Theorem 5.1

Having noted the relationship between the pullback families of connections arising from Levine's construction and the family $D_{U, r}$ we search for natural bundle maps whose families of pushforward connections are related to the family $D_{Q, r}$ on $Q$.

Pushforward at zero. Let $\pi: E \rightarrow Q$ be the atomic bundle map over $\mathbb{P}(E \oplus F)$, defined by $\pi(e)=[e, 0]$, where $[e, f]$ denotes the image of $(e, f)$ in $Q$. Note that $\operatorname{Div}(\pi)=[X]$ since $U=E$ on $X$. 
Theorem 5.8. Let $0<r<1$ and $0<s<\infty$ be related by $r=s /(1+s)$. Then

$$
D_{Q, r}=\vec{D}_{\pi, s} \quad \text { on } Q \text { over } \mathbb{P}(E \oplus F),
$$

where $D_{Q, r}$ is defined by $(2.20)$.

\section{Corollary 5.9.}

$$
\phi\left(\left(\vec{D}_{\pi, 0}\right)\right)=\phi\left(\left(\vec{D}_{\alpha, 0}\right)\right) \quad \text { on } \mathbb{P}(E \oplus F) .
$$

Proof of Theorem 5.8. Let $\psi: F \rightarrow Q$ be defined by $\psi(f)=[0, f]$. We need to show that $\psi^{-1} \circ \vec{D}_{\pi, s} \circ \psi=\vec{D}_{\alpha, r}$ on $F$ over $\operatorname{Hom}(E, F)$. Now $\pi=-\psi \alpha$ and $\psi^{*}=\left(1-\alpha \alpha^{*} /\left(1+|\alpha|^{2}\right)\right) \psi^{-1}$. So $\pi^{*}=-\alpha^{*} \psi^{-1} /\left(1+|\alpha|^{2}\right)$. Now follow your nose.

Pushforward at infinity. In analogy with the previous three cases, we consider the map $\pi: \mathbf{L} \rightarrow \mathbf{Q}$ over $\widehat{\mathbb{P}}(E \oplus F)$ given by $\mathbf{L} \hookrightarrow \mathbf{F} \stackrel{\psi}{\longrightarrow} \mathbf{Q}$. Since $\mathbf{L}=\mathbf{U}$ on $\mathbb{P}(F), \pi$ vanishes on the codimension 2 submanifold $\mathbb{P}(F)$ and so is not atomic unless rank $F=1$. Furthermore, $\vec{D}_{\pi, s} \neq D_{\mathbf{Q}, r}$ unless rank $F=1$. Locally, however, $\pi$ is of the form $(t, 0, \ldots, 0)$, where $t$ is an atomic $\mathbb{C}$-valued function, and so there is still some hope that the Chern current $\phi\left(\left(\vec{D}_{\pi, 0}\right)\right)$ of the degenerately vanishing map $\pi$ exists.

Theorem 5.10. Let $0<s<\infty$ and $\infty>r>1$ be related by $r=(1+s) / s$, and let $\omega(s)$ be the global $\operatorname{Hom}(\mathbf{Q}, \mathbf{Q})$-valued 1-form defined over $\widehat{\mathbb{P}}(E \oplus F)$ by $\omega(s):=\vec{D}_{\pi, s}-D_{\mathbf{Q}, r}$. Then in a local fibre variable $u$ on $\operatorname{Hom}(E, F)$,

$$
\omega(s)=-\tilde{\chi}_{s} \frac{u^{*} D u}{|u|^{2}}(1-P) \quad \text { on } \operatorname{Hom}(E, F) \sim X,
$$

where $\widetilde{\chi}_{s}:=1 /\left(1+s+s|u|^{2}\right)$ and $P:=u^{*} u /|u|^{2}$. In particular, when $\operatorname{rank} F=1$,

$$
\vec{D}_{\pi, s}=D_{\mathbf{Q}, r} \quad \text { on } \mathbf{Q} \text { over } \widehat{\mathbb{P}}(E \oplus F) .
$$

Proof. Let $e, f$ be local frames for $E, F$. Then $\ell=\alpha(e)$ and $q=[0, f]$ are local frames for $L$ and $Q$ over $\operatorname{Hom}(E, F)$. Define $u$ and $w$ by $\alpha(e)=u f$ and $\pi(\ell)=w q$. Let $\vec{\omega}_{\alpha, r}$ and $\vec{\omega}_{\pi, s}$ be the corresponding local gauges for $\vec{D}_{\alpha, r}$ and $\vec{D}_{\pi, s}$. Recall that

$$
\omega_{Q}=\vec{\omega}_{\alpha, 1}=\omega_{F}-\frac{u^{*} D u}{|u|^{2}+1} \quad \text { and } \quad \omega_{L}=\overleftarrow{\omega}_{\alpha, 0}=\omega_{E}+\frac{D u u^{*}}{|u|^{2}}
$$


Then

$$
\begin{aligned}
\omega(s) & =\vec{\omega}_{\pi, s}-\vec{\omega}_{\alpha, r}=\omega_{Q}-\frac{w^{*} D w}{|w|^{2}+s}-\omega_{F}+\frac{u^{*} D u}{|u|^{2}+r} \\
& =-\frac{u^{*} D u}{|u|^{2}+1}+\frac{u^{*} D u}{|u|^{2}+r}-\frac{w^{*} D w}{|w|^{2}+s} .
\end{aligned}
$$

Now $w=u, w^{*}=u^{*} /|u|^{2}\left(|u|^{2}+1\right)$ and $|w|^{2} /\left(|w|^{2}+s\right)=\widetilde{\chi}_{s}$. Substituting

$$
\frac{w^{*} D w}{|w|^{2}+s}=\frac{u^{*} D u\left((1-P)-|u|^{2} P\right)}{|u|^{2}\left(|u|^{2}+1\right)\left(1+s+s|u|^{2}\right)}
$$

into (5.12) yields (5.11).

Set $D_{s}:=\psi^{-1} \circ \vec{D}_{\pi, s} \circ \psi$ on $F$ and define

$$
D_{\sigma}:=\sigma D_{s}+(1-\sigma) \vec{D}_{\alpha, r} \quad \text { for } 0<\sigma<1 \text { on } F
$$

Let $\Omega_{\alpha, r}, \Omega_{s}, \Omega_{\sigma}$ be the local curvature matrices of the connections $\vec{D}_{\alpha, r}, D_{s}$, $D_{\sigma}$.

Proposition 5.13. Let $\chi_{r}=|u|^{2} /|u|^{2}+r=s|u|^{2} / 1+s+s|u|^{2}$ and $\Lambda=$ $-D u^{*} D u /|u|^{2}$. Then, on $\operatorname{Hom}(E, F) \sim X$,

$$
\Omega_{\sigma}=\sigma\left(\Omega_{s}-\Omega_{\alpha, r}\right)+\Omega_{\alpha, r}
$$

where

$$
\Omega_{\alpha, r}=\left(1-\chi_{r} P\right)\left(\Omega_{F}+\chi_{r} \Lambda\right)+\chi_{r} P \Omega_{E}
$$

and

$$
\Omega_{s}=\Omega_{\alpha, r}+\tilde{\chi}_{s}\left(\Omega_{0}-\Omega_{F}-\chi_{r} P \Lambda(1-P)\right) .
$$

Here,

$$
\Omega_{0}=\Omega_{F}+(1-P) \Lambda(1-P)+P(\beta-\lambda)-P \Omega_{F}(1-P),
$$

where $\lambda=\operatorname{Tr}(\Lambda)=D u D u^{*} /|u|^{2}$ and $\beta=D u u^{*} u D u^{*} /|u|^{2}$. 


\section{Corollary 5.18.}

$$
\operatorname{Tr}\left(\Omega_{s}\right)=\operatorname{Tr}\left(\Omega_{\alpha, r}\right) .
$$

Consequently, the Chern current $c_{1}^{m}\left(\left(\vec{D}_{\pi, 0}\right)\right)$ exists and equals

$$
c_{1}^{m}\left(\left(\vec{D}_{\pi, 0}\right)\right)=c_{1}^{m}\left(\left(\vec{D}_{\alpha, \infty}\right)\right) \quad \text { on } \widehat{\mathbb{P}}(E \oplus F) .
$$

More generally, let

$$
T_{s}(\phi):=\int_{0}^{1} \phi\left(\omega(s) ; \Omega_{\sigma}\right) d \sigma
$$

Then

$$
\phi\left(\vec{D}_{\pi, s}\right)-\phi\left(\vec{D}_{\alpha, r}\right)=d T_{s}(\phi) \quad \text { on } \widehat{\mathbb{P}}(E \oplus F) .
$$

Proposition 5.21. $\quad T_{s}\left(c_{2}\right) \rightarrow T_{0}\left(c_{2}\right)$ in $L_{\mathrm{loc}}^{1}(\widehat{\mathbb{P}}(E \oplus F))$ as $s \rightarrow 0$. In local homogeneous coordinates $[t, u]$, for $\mathbb{P}(E \oplus F)$,

$$
T_{0}\left(c_{2}\right)=-\frac{D u \Omega_{F} u^{*}}{|u|^{2}}+\frac{u \Omega_{F} u^{*}}{|u|^{2}} \frac{D u u^{*}}{|u|^{2}} \in C^{\infty}(\mathbb{P}(E \oplus F) \sim X),
$$

and so the Chern current $c_{2}\left(\left(\vec{D}_{\pi, 0}\right)\right)$ exists and equals

$$
c_{2}\left(\left(\vec{D}_{\pi, 0}\right)\right)=c_{2}\left(\left(\vec{D}_{\alpha, \infty}\right)\right)+d T_{0}\left(c_{2}\right) .
$$

In particular, the singular parts of the two Chern currents are equal.

Proposition 5.22. Suppose that $D_{E}$ and $D_{F}$ are flat connections. Then for any invariant polynomial $\phi$,

$$
T_{s}(\phi) \rightarrow 0 \quad \text { in } L_{\text {loc }}^{1}(\widehat{\mathbb{P}}(E \oplus F)) \quad \text { as } s \rightarrow 0 .
$$

Consequently, if $\phi$ is of pure degree $N$, then

$$
\phi\left(\left(\vec{D}_{\pi, 0}\right)\right)=\phi\left(\left(\vec{D}_{\alpha, \infty}\right)\right)=\left(-c_{1}\left(D_{L}\right)\right)^{N-1}\left[\mathbb{P}(F)_{\infty}\right] \quad \text { on } \widehat{\mathbb{P}}(E \oplus F) .
$$

Remark 5.23. In the nonflat case $T_{s}\left(c_{3}\right)$ does not converge in $L_{\text {loc }}^{1}$ to $T_{0}\left(c_{3}\right)$ (see Example 5.26 below). Most likely it converges to a current of the form $T=\omega+\eta\left[\mathbb{P}(F)_{\infty}\right]$, where $\omega \in C^{\infty}\left(\widehat{\mathbb{P}}(E \oplus F) \sim \mathbb{P}(F)_{\infty}\right) \cap L_{\text {loc }}^{1}(\widehat{\mathbb{P}}(E \oplus F))$ and $\eta$ is a degree $3 L_{\text {loc }}^{1}$ form on $\mathbb{P}(F)_{\infty}$. Therefore, the Chern current $c_{3}\left(\left(\vec{D}_{\pi, 0}\right)\right)$ will still exist though it is impossible to predict the precise form of its singular part without much calculation! 
Proof of Proposition 5.13. Simplifying [HL, III.2.3] in the case that $\chi$ is the algebraic approximation mode gives

$$
\Omega_{\alpha, r}=\left(1-\chi_{r} P\right)\left(\Omega_{F}+\chi_{r} \Lambda\right)+\chi_{r} P \Omega_{E},
$$

which can be rewritten as

$$
\Omega_{\alpha, r}=\chi_{r} \Omega_{\alpha, 0}+\left(1-\chi_{r}\right)\left(\Omega_{F}+\chi_{r} P \Lambda\right)
$$

where

$$
\Omega_{\alpha, 0}=(1-P)\left(\Omega_{F}+\Lambda\right)+P \Omega_{E} .
$$

Replacing $\left(\alpha, u, \Omega_{F}, \Omega_{E}\right)$ by $\left(\pi, w, \Omega_{Q}, \Omega_{L}\right)$ in (5.24) and (5.25) and simplifying yields (5.16) and (5.17).

Proof of Proposition 5.21. Recall that $c_{2}=\frac{1}{2}\left(c_{1}^{2}-b_{2}\right)$. Let $\alpha=$ $D u u^{*} /|u|^{2}$ and note that $\alpha^{2}=0$. Plugging (5.11) and (5.14) into (5.19) gives

$$
T_{s}\left(b_{2}\right)=\tilde{\chi}_{s} \chi_{r} \alpha \lambda+\tilde{\chi}_{s}\left(\frac{u \Omega_{F} u^{*}}{|u|^{2}} \alpha-\frac{D u \Omega_{F} u^{*}}{|u|^{2}}\right) .
$$

Since

$$
u \frac{\partial}{\partial u}\left\llcornerT _ { s } ( b _ { 2 } ) = \tilde { \chi } _ { s } \chi _ { r } ( \lambda - \beta ) \quad \text { and } \quad u ^ { * } \frac { \partial } { \partial u ^ { * } } \left\llcorner T_{s}\left(b_{2}\right)=0,\right.\right.
$$

the expression for $T_{s}\left(b_{2}\right)$ in homogeneous coordinates is

$$
T_{s}\left(b_{2}\right)_{H C}=T_{s}\left(b_{2}\right)-\widetilde{\chi}_{s} \chi_{r}(\lambda-\beta) \frac{d t}{t} .
$$

Since $\tilde{\chi}_{s} \chi_{r}$ is bounded by 1 and converges to zero a.e. as $s \rightarrow 0$ and $(\lambda-$ $\beta)(d t / t) \in L_{\text {loc }}^{1}(\widehat{\mathbb{P}}(E \oplus F))$, the Lebesgue Dominated Convergence Theorem implies that $T_{s}\left(b_{2}\right) \rightarrow T_{0}\left(b_{2}\right)$ in $L_{\text {loc }}^{1}(\widehat{\mathbb{P}}(E \oplus F))$ as $s \rightarrow 0$.

Example 5.26. The expression for $T_{s}\left(b_{3}\right)$ in homogeneous coordinates includes the term

$$
\frac{s|u|^{2}(1+s)|t|^{2}}{\left((1+s)|t|^{2}+s|u|^{2}\right)^{3}} \frac{D u(1-P) \Omega_{F} u^{*}}{|u|^{2}} d t d \bar{t}
$$

which converges to zero a.e., but not in $L_{\text {loc }}^{1}$. 
Proof of Proposition 5.22. We can assume that $\phi=\prod_{j=1}^{m} b_{k_{j}}$. First use the fact that $\alpha^{2}=0$ to obtain the recursion formula,

$$
\operatorname{Tr}\left(\omega(s) \Omega_{\sigma}^{k-1}\right)=-\sigma \widetilde{\chi}_{s} \lambda \operatorname{Tr}\left(\omega(s) \Omega_{\sigma}^{k-2}\right)+(-1)^{k} \tilde{\chi}_{s} \chi_{r}\left(\sigma \widetilde{\chi}_{s}+\chi_{r}\right)^{k-2} \alpha \lambda^{k-1} .
$$

Therefore, $b_{k}\left(\omega(s) ; \Omega_{\sigma}\right)=\chi_{r} C_{1}(s, \sigma, k) \alpha \lambda^{k-1}$ where $C_{1}(s, \sigma, k) \leq C$. Furthermore, since $b_{k}\left(\Omega_{\sigma}\right)=C_{2}(s, \sigma, k) \lambda^{k} \bmod \alpha$, where $C_{2}(s, \sigma, k) \leq C$ and the typical term of $\phi\left(\omega(s) ; \Omega_{\sigma}\right)$ is $b_{k_{1}}\left(\omega(s) ; \Omega_{\sigma}\right) \prod_{j=2}^{m} b_{k_{j}}\left(\Omega_{\sigma}\right)$, we have that

$$
T_{s}(\phi)=C_{3}(s, k) \alpha \lambda^{N-1},
$$

where $N=\operatorname{deg} \phi$ and $C_{3}(s, k) \leq C$ with $C_{3}(s, k) \rightarrow 0$ as $s \rightarrow 0$. So since $\partial / \partial u^{*}\left\llcorner T_{s}(\phi)=0\right.$, we can conclude as before that $T_{s}(\phi) \rightarrow 0$ as $s \rightarrow 0$ in $L_{\text {loc }}^{1}(\widehat{\mathbb{P}}(E \oplus F))$.

6. Meromorphic quaternionic line bundle homomorphisms. The theory of Chern currents associated with endomorphisms of smooth quaternionic line bundle maps has strong analogies with that of maps between complex line bundles (see [HL, II,V]). In this section we study the Chern currents associated with meromorphic maps between quaternionic line bundles. As we shall see the analytic aspects of this case are much more delicate than in the complex case.

First, we review Harvey and Lawson's results in the complex case. A meromorphic section $\nu$ of a complex line bundle $F \rightarrow X$ is defined to be one which can be expressed in each local frame $f$ for $F$ as $\nu=(a / b) f$ where $a, b$ are smooth complex valued functions on $X$. Such a section is called atomic if each of the local functions $a, b$ are atomic. The divisor of $\nu$ is defined by $\operatorname{Div}(\nu)=\operatorname{Div}(a)-\operatorname{Div}(b)$. These notions are well defined since if $g, h: X \rightarrow \mathbb{C}$ are atomic, then $g h$ is atomic and $\operatorname{Div}(g h)=\operatorname{Div}(g)+\operatorname{Div}(h)$. Fix a connection $D_{F}$ on $F$. The 1 -form $\tau:=d a / a-d b / b+\omega_{F}$ is a globally defined $L_{\text {loc }}^{1}$ form on $X$ and we have the current equation,

$$
\frac{1}{2 \pi i} d \tau=\operatorname{Div}(\nu)-c_{1}\left(D_{F}\right)
$$

on $X$.

Now we turn to the quaternionic case. Let $E, F$ be quaternionic line bundles over a manifold $X$.

Definition 6.1. A meromorphic quaternion-linear bundle map $\nu$ from $E$ to $F$ is a map which can be expressed in each pair of local frames $e$ for $E$ and $f$ for $F$ as

$$
\nu e=a b^{-1} f,
$$


where $a$ and $b$ are smooth quaternion valued local functions on $X$. Such a mapping $\nu$ is called atomic if each of the local $\mathbb{R}^{4}$-valued functions $a$ and $b$ are atomic.

We would like to define the divisor of such an atomic map $\nu$ locally by the current equation,

$$
\operatorname{Div}(\nu)=\operatorname{Div}(a)-\operatorname{Div}(b) .
$$

The only difficulty in proving the quaternionic analogues of the results described above is to show that the $\operatorname{divisor} \operatorname{Div}(\nu)$ is a well defined global current on $X$. We will discuss this problem in more generality later, but first we restrict our attention to a simple class of atomic meromorphic quaternionic maps from $E$ to $F$ for which it is easy to show that the divisor is well defined. This class consists of all maps $\nu$ for which the union $Z$ of the zero sets of all the local functions $a$ defined by (6.2) is disjoint from the union $P$ of the zero sets of the local functions $b$. If this is the case we say that $\nu$ is a determined meromorphic map and call $Z$ and $P$ the zero and pole sets of $\nu$. Note that this class of meromorphic maps includes all those maps which can be defined by a section of the bundle $\mathbb{P}_{\mathbb{H}}(E \oplus F) \rightarrow X$ of quaternionic projective spaces.

Proposition 6.4. Let $\nu$ be a determined meromorphic quaternion linear bundle map between quaternionic line bundles $E$ and $F$. Suppose that $\nu$ is atomic. Then the divisor, $\operatorname{Div}(\nu)$, of $\nu$ defined by (6.3) is a well defined current on $X$.

Next we state a generalization of Harvey and Lawson's main theorem on homomorphisms between quaternionic line bundles, [HL, V.2.26, 36]. Endow $E$ and $F$ with metrics with respect to which scalar multiplication by unit quaternions is a pointwise isometry. Choose metric compatible connections $D_{E}$ and $D_{F}$ on $E$ and $F$ with respect to which scalar multiplication by any quaternion is parallel. The instanton form, $u\left(D_{F}\right)$, is defined by

$$
u\left(D_{F}\right):=\frac{1}{16 \pi^{2}} \operatorname{tr}\left(\Omega_{F}^{2}\right) .
$$

We use the notation $e:=u\left(D_{E}\right)$ and $f:=u\left(D_{F}\right)$.

Theorem 6.5. Let $\nu$ be an atomic determined meromorphic quaternionic bundle map from $E$ to $F$. Then there is a canonical $L_{\text {loc }}^{1}$ form $T$ on $X$ called the instanton transgression current such that

$$
f-e-\operatorname{Div}(\nu)=d T
$$

as currents on $X$, where

$$
16 \pi^{2} T=-\operatorname{tr}\left(\tau R_{E}\right)-\operatorname{tr}\left(\tau^{\prime} R_{F}\right)+\frac{1}{3} \operatorname{tr}\left(\tau^{3}\right) .
$$

Here, $\tau=\nu^{-1} \circ D \nu=\nu^{-1} \circ D_{F} \circ \nu-D_{E}$ and $\tau^{\prime}=D \nu \circ \nu^{-1}=D_{F}-\nu \circ D_{E} \circ$ $\nu^{-1}$ are $L_{\text {loc }}^{1}$ forms on $X$ with values in $\operatorname{End}_{\mathbb{H}}(E)$ and $\operatorname{End}_{\mathbb{H}}(F)$ respectively, and $R_{E}$ and $R_{F}$ are the curvature operators on $E$ and $F$. 
Remark 6.7. Suppose that $\nu$ is a section of the bundle $\mathbb{P}_{\mathbb{H}}(E \oplus F)$ of quaternionic projective spaces and let $E \stackrel{\alpha}{\rightarrow} F$ be the bundle homomorphism induced by $\nu$ over $X \sim P$. Just as in the complex case, the pullback family of connections on $E$ defined by $\overleftarrow{D}_{s}:=D_{E}+\chi_{s} \alpha \circ D \alpha$ using the algebraic approximation mode, $\chi(t)=t /(1+t)$, is compactified by the family of connections $\nu^{*}\left(D_{U, s}\right)$ on the pullback $\nu^{*}(U)$ of the tautological quaternionic line bundle $U \rightarrow \mathbb{P}_{\mathbb{H}}(E \oplus F)$. Furthermore, the current limit of the corresponding smooth transgression formula

$$
u\left(\overleftarrow{D}_{r}\right)-u\left(\overleftarrow{D}_{s}\right)=d T_{r, s}
$$

on $X$

is the current equation

$$
e-f+\operatorname{Div}(\nu)=-d T
$$

of Theorem 6.5.

The proof of Proposition 6.4 relies on the following fundamental lemma.

Lemma 6.8. Let $a, b: X \rightarrow \mathbb{H}$ be atomic. Suppose that the zero sets $A$, $B$ of $a, b$ are disjoint. Then

(1) ab is atomic.

(2) Let $\tau(b)=d b b^{-1}, \tau^{\prime}(a)=a^{-1} d a$ and set

$$
\Omega=\frac{1}{2} \operatorname{Re}\left(\tau^{\prime}(a) \tau(b)\right)=\frac{1}{2} \operatorname{Re}\left(\frac{\bar{a} d a d b \bar{b}}{|a|^{2}|b|^{2}}\right) .
$$

Then the smooth forms $\Omega$ and $d \Omega$ on $X \sim(A \cup B)$ have (unique) $L_{\mathrm{loc}}^{1}$ extensions $\tilde{\Omega}$ and $\widetilde{d \Omega}$ to all of $X$. Furthermore,

$$
d \tilde{\Omega}=\widetilde{d \Omega} \quad \text { in } L_{\mathrm{loc}}^{1}(X) .
$$

(3) Let $\vartheta(f):=f^{*} \vartheta \in L_{\text {loc }}^{1}(X)$ denote the pullback of the solid angle kernel $\vartheta$ on $\mathbb{H}$ to $X$ via an atomic function $f$. Then

$$
\vartheta(a b)=\vartheta(a)+\vartheta(b)+d \tilde{\Omega} \quad \text { in } L_{\mathrm{loc}}^{1}(X) .
$$

(4) $\operatorname{Div}(a b)=\operatorname{Div}(a)+\operatorname{Div}(b)$ on $X$.

(5) (2) holds with $b$ replaced by $b^{-1}$.

(6) The smooth form $\vartheta\left(a b^{-1}\right)$ on $X \sim(A \cup B)$ has an $L_{\text {loc }}^{1}$ extension to all of $X$ and

$$
\vartheta\left(a b^{-1}\right)=\vartheta(a)-\vartheta(b)+d \tilde{\Omega} \quad \text { in } L_{\mathrm{loc}}^{1}(X),
$$

where $\Omega=\frac{1}{2} \operatorname{Re}\left(\tau^{\prime}(a) \tau^{\prime}(b)\right)$. In particular,

$$
\vartheta\left(b^{-1}\right)=-\vartheta(b) .
$$


Proof.

(1) We need to show that $d(a b)^{I} /|a b|^{p} \in L_{\text {loc }}^{1}(X)$ for all $I$ such that $p=$ $|I| \leq 3$. Now each such form is dominated by sums of terms of the form $\left(d a^{J} /|a|^{j}\right)\left(d b^{K} /|b|^{k}\right)$, where $j=|J| \leq 3, k=|K| \leq 3$. But all of these forms belong to $L_{\text {loc }}^{1}(X)$ since the zero sets of $a$ and $b$ are disjoint and, in a neighbourhood of the zero set of $a, d b^{K} /|b|^{k}$ is bounded while $d a^{J} /|a|^{j} \in L_{\text {loc }}^{1}(X)$.

(2) Similarly, $\Omega$ and $d \Omega$ have unique $L_{\text {loc }}^{1}$ extensions $\tilde{\Omega}$ and $\widetilde{d \Omega}$ to all of $X$. Let $\chi_{s}=\left(|a|^{2} /\left(s^{2}+|a|^{2}\right)\right)\left(|b|^{2} /\left(s^{2}+|b|^{2}\right)\right)$. Using the atomicity of $a$ and $b$ to dominate $d \chi_{s} \Omega$ by an $s$-independent $L_{\text {loc }}^{1}$ form and applying the Lebesgue Dominated Convergence Theorem three times gives

$$
d \tilde{\Omega}=d\left(\lim _{s \rightarrow 0} \chi_{s} \Omega\right)=\lim _{s \rightarrow 0} d \chi_{s} \Omega+\lim _{s \rightarrow 0} \chi_{s} d \Omega=0+\widetilde{d \Omega} .
$$

(3) Let $x$ be a coordinate on $\mathbb{H}$ and set $\tau=d x x^{-1}$ and $\tau^{\prime}=x^{-1} d x$. Then (see [HL, V.2.34]) the solid angle kernel $\vartheta$ on $\mathbb{H}$ is given by

$$
\vartheta=-\frac{1}{6} \operatorname{Re}\left(\tau^{3}\right) .
$$

Let $\tau(f):=f^{*} \tau$ and $\tau^{\prime}(f):=f^{*} \tau^{\prime}$ for any function $f: X \rightarrow \mathbb{H}$, and note that

$$
\tau(a b)=\tau(a)+a \tau(b) a^{-1} .
$$

Then

$$
\vartheta(a b)=-\frac{1}{6} \operatorname{Re}\left(\tau^{3}(a b)\right)=\vartheta(a)+\vartheta(b)+\frac{1}{2} \operatorname{Re}\left(d \tau^{\prime}(a) \tau(b)-\tau^{\prime}(a) d \tau(b)\right),
$$

since $\tau^{2}=d \tau$ and $\left(\tau^{\prime}\right)^{2}=-d \tau^{\prime}$. So we have the equation of smooth forms

$$
\vartheta(a b)=\vartheta(a)+\vartheta(b)+d \Omega \quad \text { on } X \sim(A \cup B) .
$$

Therefore,

$$
\widetilde{d \Omega}=\vartheta(a b)-\vartheta(a)-\vartheta(b)
$$

in $L_{\text {loc }}^{1}(X)$,

since it is true almost everywhere. (3) now follows from (2) and (4) from (3).

Proof of Proposition 6.4. Suppose that $\nu e=a b^{-1} f$ and $\nu e^{\prime}=c d^{-1} f^{\prime}$ where $e^{\prime}=g e$ and $f^{\prime}=h f$. Since $g a b^{-1}=c d^{-1} h$ with $g$ and $h$ nonzero, Lemma 6.8(6) implies that

$$
\vartheta(g a)-\vartheta(b)+d \tilde{\Omega}\left(g a, b^{-1}\right)=\vartheta(c)-\vartheta\left(h^{-1} d\right)+d \tilde{\Omega}\left(c, h^{-1} d\right) .
$$

Combining this with Lemma 6.8(4) gives $\operatorname{Div}(a)-\operatorname{Div}(b)=\operatorname{Div}(g a)-\operatorname{Div}(b)=$ $\operatorname{Div}(c)-\operatorname{Div}\left(h^{-1} d\right)=\operatorname{Div}(c)-\operatorname{Div}(d)$ as required. 
Proof of Theorem 6.5. We check that the smooth form $T$, defined on $X \sim(Z \cup P)$ by (6.6), has an $L_{\text {loc }}^{1}$ extension $\tilde{T}$ to all of $X$ and compute $d \tilde{T}$. Write $\nu e=a b^{-1} f$. Then $T$ is a sum of terms, each of which is the product of an $L_{\text {loc }}^{\infty}$ form on $X$ with one of the forms

$$
\frac{d a^{I} d b^{J}}{|a|^{p}|b|^{q}} \quad \text { where } p=|I|, q=|J| \text { and } p+q \leq 3 .
$$

But each of these forms is in $L_{\text {loc }}^{1}(X)$ since $a$ and $b$ are atomic and have disjoint zero sets. Therefore, $T$ has an $L_{\text {loc }}^{1}$ extension $\tilde{T}$ to all of $X$. Next note that the smooth form $d T$ on $X \sim(Z \cup P)$ has a $C^{\infty}$ extension $\widetilde{d T}$ to all of $X$ since, by [HL, V.2.10], $d T=u\left(D_{F}\right)-u\left(D_{E}\right)$ on $X \sim(Z \cup P)$. Define $S:=\widetilde{d T}-\widetilde{d T}$ on $X$. Since $\widetilde{d T}=u\left(D_{F}\right)-u\left(D_{E}\right)$, we just need to check that $S=-\operatorname{Div}(\nu)$. Let $T_{\text {top }}$ be that part of $\tilde{T}$ which is of top degree 3 in the differentials $d a_{i}, d b_{j}$ and let $T^{\prime}:=\tilde{T}-T_{\text {top }}$. Then, since trace equals four times the real part,

$$
T_{\text {top }}=\frac{1}{48 \pi^{2}} \operatorname{tr}\left(\tau^{3}\left(a b^{-1}\right)\right)=-\frac{1}{2 \pi^{2}} \vartheta\left(a b^{-1}\right),
$$

and so, by Lemma $6.8(6), d T_{\text {top }}=-(\operatorname{Div}(a)-\operatorname{Div}(b))=-\operatorname{Div}(\nu)$. Finally, since $d T^{\prime}$ is $L_{\text {loc }}^{1}$ and $\widetilde{d T}+S=d T^{\prime}-\operatorname{Div}(\nu)$ we are forced to conclude that $S=-\operatorname{Div}(\nu)$, as required.

Let $a, b: X \rightarrow \mathbb{H}$ be atomic. Ideally, we would like to prove that $a b$ is atomic and that $\operatorname{Div}(a b)=\operatorname{Div}(a)+\operatorname{Div}(b)$. This has not been possible. Neither have we found any counterexamples. Instead we describe some extra assumptions which can be placed on the pair $a, b$ to ensure that the desired conclusions hold. Proofs are left to the reader. The first result is motivated by [HS, 3.2].

Definition 6.9 ([HS, 3.2]). Let $u$ be a smooth $\mathbb{R}^{n}$-valued function on $X^{\text {open }} \subset \mathbb{R}^{m}$. We say that $u$ vanishes algebraically if, for each compact set $K \subset X$, there exist positive constants $c$ and $N$ such that

$$
|u(x)| \geq c \operatorname{dist}(x, \operatorname{Zero}(u))^{N} \quad \text { for all } x \in K .
$$

The zero set of $u$ is said to have strong codimension greater than $n-1$ if for each compact $K \subset X$ there exists an $\varepsilon>0$ such that the upper Minkowski content of Zero $(u) \cap K$ in dimension $m-n+1-\varepsilon$ is finite.

Note. Harvey and Semmes proved that if $u$ vanishes algebraically and if its zero set has strong codimension greater than $n-1$, then $u$ is atomic. 
Proposition 6.10. Let $a, b: X \rightarrow \mathbb{H}$ be smooth.

(1) If $a$ and $b$ vanish algebraically, then so does $a b$.

(2) If the zero sets of $a$ and $b$ have strong codimension greater than 3 , then so does that of $a b$.

(3) Therefore, if the hypotheses of (1) and (2) hold, then $a, b$ and ab are all atomic.

Corollary 6.11. Let $a, b: X \rightarrow \mathbb{H}$ be real analytic and suppose that each irreducible component of the zero sets of $a$ and $b$ has codimension $\geq 4$. Then $a$, $b$ and $a b$ are all atomic.

Let $\mathcal{H}^{k}$ denote $k$-dimensional Hausdorff measure. The following result follows from the Federer Support Theorem for flat currents (see $[F]$ ).

Proposition 6.12. Let $a, b: X \rightarrow \mathbb{H}$ be atomic. Let $A, B$ denote the zero sets of $a, b$ and let $n:=\operatorname{dim} X$. Suppose that $a b$ is atomic and that either

(1) $\mathcal{H}^{n-3}(A \cap B)=0$ and $\Omega=\frac{1}{2} \operatorname{Re}\left(\bar{a} d a d b \bar{b} /|a|^{2}|b|^{2}\right)$ has an $L_{\text {loc }}^{1}$ extension $\tilde{\Omega}$ to all of $X$, or

(2) $\mathcal{H}^{n-4}(A \cap B)=0$.

Then,

$$
\operatorname{Div}(a b)=\operatorname{Div}(a)+\operatorname{Div}(b) \quad \text { on } X .
$$

Note. In contrast to this note that if $a: X \rightarrow \mathbb{H}$ is atomic, then $a^{n}$ is atomic and $\operatorname{Div}\left(a^{n}\right)=n \operatorname{Div}(a)$ on $X$.

We conclude this section with an example of a pair of polynomial functions $a$ and $b$ which do not satisfy the hypotheses of Lemma 6.8 or Proposition 6.12. Nevertheless, we can compute directly that $\operatorname{Div}(a b)=\operatorname{Div}(a)+\operatorname{Div}(b)$. One obvious way to try to prove that $\operatorname{Div}(a b)=\operatorname{Div}(a)+\operatorname{Div}(b)$ for any pair of atomic functions $a$ and $b$ for which $a b$ is also atomic would be to find a natural current extension $\tilde{\Omega}$ to all of $X$ of the smooth form $\Omega$ on $X \sim(A \cup B)$ and to show that $d \tilde{\Omega}=\vartheta(a b)-\vartheta(a)-\vartheta(b)$. The example below shows that we cannot hope to extend $\Omega$ to an $L_{\text {loc }}^{1}$ form on all of $X$. We have not been able to find a natural current extension of $\Omega$. Neither have we been able to find a pair of functions $a, b$ for which $\operatorname{Div}(a b) \neq \operatorname{Div}(a)+\operatorname{Div}(b)$. 
Example 6.13. Let $a, b: \mathbb{H} \rightarrow \mathbb{H}$ be defined by

$$
a\left(x_{0}, x_{1}, x_{2}, x_{3}\right)=\left(x_{0}, x_{1}^{3}, x_{2}^{3}, x_{3}^{3}\right) \quad \text { and } \quad b\left(x_{0}, x_{1}, x_{2}, x_{3}\right)=\left(x_{0}, x_{1}, x_{2}, x_{3}\right) .
$$

Then,

(1) $a, b$ and $a b$ are all atomic,

(2) $\mathcal{H}^{0}(A \cap B)=1>0$,

(3) $\Omega \notin L_{\mathrm{loc}}^{1}(\mathbb{H})$, but

(4) $\operatorname{Div}(a)=[0], \operatorname{Div}(b)=[0]$ and $\operatorname{Div}(a b)=2[0]$, so that

$$
\operatorname{Div}(a b)=\operatorname{Div}(a)+\operatorname{Div}(b) \quad \text { on } \mathbb{H} \text {. }
$$

Acknowledgements. The results in this paper are contained in the author's thesis [Z1]. I would like to thank my advisor Reese Harvey for suggesting this problem, for his many helpful ideas and comments, and for being a huge source of personal inspiration. Thanks also to Blaine Lawson and Stephen Semmes for their interest and encouragement. This research was supported by a fellowship from the Schlumberger Foundation.

\section{REFERENCES}

[B] R. BotT, A residue formula for holomorphic vector fields, J. Differential Geom. 1 (1967), 311-330.

[BC] R. BotT \& S. S. CheRn, Hermitian vector bundles and the equidistribution of the zeros of their holomorphic sections, Acta. Math. 114 (1968), 71-112.

[F] H. Federer, Geometric Measure Theory, Springer-Verlag, New York, 1969.

[GS] H. Gillet \& C. SoulÉ, Characteristic classes for algebraic vector bundles with Hermitian metrics II, Ann. of Math. 131 (1990), 205-238.

[HL] F. R. HARvey $\&$ H. B. LAWson, A theory of characteristic currents associated with a singular connection, Astérisque 213 (1993), 1-268.

[HL-A] _ A theory of characteristic currents associated with a singular connectionAnnouncement, Bull. Amer. Math. Soc. 31 (1994), 54-63.

[HS] F. R. HARvey \& S. Semmes, Zero divisors of atomic functions, Ann. Math. 135 (1992), 567-600.

[LM] H. B. Lawson \& M. Michelsohn, Spin Geometry, Princeton University Press, Princeton, New Jersey, 1989.

[L] H. LEVINE, A theorem on holomorphic mappings into complex projective space, Ann. of Math. 71 (1960), 529-535.

[Z1] J. ZWECK, Compactification problems in the theory of characteristic currents associated to a singular connection, Thesis, Rice University (1993).

[Z2] - Euler and Pontrjagin currents of a section of a compactified real bundle, Differential Geom. Appl. 1-33, (to appear). 
Department of Mathematics Rice University

Houston, Texas 77005

CurRent ADDREss:

Department of Mathematics

University of Nevada, Reno

Reno, Nevada 89557

E-MAIL: zweck@math.unr.edu

Received: March 24th, 1994. 